\title{
LEARNING MIXTURES OF PRODUCT DISTRIBUTIONS OVER DISCRETE DOMAINS*
}

\author{
JON FELDMAN ${ }^{\dagger}$, RYAN O’DONNELL ${ }^{\ddagger}$, AND ROCCO A. SERVEDIO $§$
}

\begin{abstract}
We consider the problem of learning mixtures of product distributions over discrete domains in the distribution learning framework introduced by Kearns et al. [Proceedings of the 26th Annual Symposium on Theory of Computing (STOC), Montréal, QC, 1994, ACM, New York, pp. 273-282]. We give a poly $(n / \epsilon)$-time algorithm for learning a mixture of $k$ arbitrary product distributions over the $n$-dimensional Boolean cube $\{0,1\}^{n}$ to accuracy $\epsilon$, for any constant $k$. Previous polynomial-time algorithms could achieve this only for $k=2$ product distributions; our result answers an open question stated independently in [M. Cryan, Learning and Approximation Algorithms for Problems Motivated by Evolutionary Trees, Ph.D. thesis, University of Warwick, Warwick, UK, 1999] and [Y. Freund and Y. Mansour, Proceedings of the 12th Annual Conference on Computational Learning Theory, 1999, pp. 183-192]. We further give evidence that no polynomial-time algorithm can succeed when $k$ is superconstant, by reduction from a difficult open problem in PAC (probably approximately correct) learning. Finally, we generalize our poly $(n / \epsilon)$-time algorithm to learn any mixture of $k=O(1)$ product distributions over $\{0,1, \ldots, b-1\}^{n}$, for any $b=O(1)$.
\end{abstract}

Key words. computational learning theory, PAC learning, mixture distributions, product distributions

AMS subject classifications. 68Q32, 68T05

DOI. $10.1137 / 060670705$

\section{Introduction.}

1.1. Framework and motivation. In this paper we study mixture distributions. Given distributions $\mathbf{X}^{1}, \ldots, \mathbf{X}^{k}$ over $\mathbf{R}^{n}$ and mixing weights $\pi^{1}, \ldots, \pi^{k}$ that sum to 1 , a draw from the mixture distribution $\mathbf{Z}$ is obtained by first selecting $i$ with probability $\pi^{i}$ and then making a draw from $\mathbf{X}^{i}$. Mixture distributions arise in many practical scientific situations as diverse as medicine, geology, and artificial intelligence; indeed, there are several textbooks devoted to the subject $[23,19]$.

Assuming that data arises as a mixture of some distributions from a class of distributions $\mathcal{C}$, it is natural to try to learn the parameters of the mixture components. Our work addresses the learning problem in the PAC-style (probably approximately correct) model introduced by Kearns et al. [18]. In this framework we are given a

* Received by the editors September 26, 2006; accepted for publication (in revised form) October 19, 2007; published electronically February 1, 2008. An extended abstract of this work appeared in the Proceedings of the 46th Annual IEEE Symposium on Foundations of Computer Science (FOCS), Pittsburgh, PA, 2005, pp. 501-510. This version contains proofs that were omitted from the extended abstract because of space limitations.

http://www.siam.org/journals/sicomp/37-5/67070.html

${ }^{\dagger}$ Google, New York Office, 76 Ninth Avenue, 4th floor, New York, NY 10011 (jonfeld@ieor. columbia.edu). Some of this author's work was done while at the Department of Industrial Engineering and Operations Research, Columbia University, supported by an NSF Mathematical Sciences Postdoctoral Research Fellowship.

${ }^{\ddagger}$ School of Computer Science, Carnegie Mellon University, 7121 Wean Hall, Pittsburgh, PA 15213 (odonnell@cs.cmu.edu). Some of this author's work was done while at the Institute for Advanced Study, supported in part by the National Science Foundation under agreement CCR-0324906. Any opinions, findings and conclusions or recommendations expressed in this material are those of the authors and do not necessarily reflect the views of the National Science Foundation.

$\S$ Department of Computer Science, Columbia University, 1214 Amsterdam Ave., Mailcode 0401, New York, NY 10027 (rocco@cs.columbia.edu). This author's research was supported in part by NSF CAREER award CCF-0347282. 
class $\mathcal{C}$ of probability distributions over $\mathbf{R}^{n}$ and access to random data sampled from an unknown mixture $\mathbf{Z}$ of $k$ unknown distributions from $\mathcal{C}$. The goal is to output a hypothesis mixture $\mathbf{Z}^{\prime}$ of $k$ distributions from $\mathcal{C}$, which (with high confidence) is $\epsilon$-close to the unknown mixture. The learning algorithm should run in time $\operatorname{poly}(n / \epsilon)$. The standard notion of "closeness" between distributions $\mathbf{Z}$ and $\mathbf{Z}^{\prime}$, proposed by Kearns et al. and used in this work, is the Kullback-Leibler $(K L)$ divergence (or relative entropy), defined as $\mathrm{KL}\left(\mathbf{Z} \| \mathbf{Z}^{\prime}\right):=\int_{x} \mathbf{Z}(x) \ln \left(\mathbf{Z}(x) / \mathbf{Z}^{\prime}(x)\right){ }^{1}$

In this paper we learn mixtures of product distributions over the Boolean cube $\{0,1\}^{n}$, and more generally over the $b$-ary cube $\{0, \ldots, b-1\}^{n}$; i.e., the classes $\mathcal{C}$ will consist of distributions $\mathbf{X}^{i}$ whose $n$ coordinates are independent distributions over $\{0,1\}$ and $\{0, \ldots, b-1\}$, respectively. ${ }^{2}$ Such learning problems have been well studied in the past, as we now describe.

1.2. Related work. In [18], Kearns et al. gave efficient algorithms for learning mixtures of Hamming balls; these are product distributions over $\{0,1\}^{n}$ in which all the coordinate means $\mathbf{E}\left[\mathbf{X}_{j}^{i}\right]$ must be either $p$ or $1-p$ for some unknown $p$ which is fixed over all mixture components. Although these algorithms can handle mixtures with $k=O(1)$ many components, the fact that the components are Hamming balls rather than general product distributions is a very strong restriction. (The algorithms also have some additional restrictions: $p$ has to be bounded away from $1 / 2$, and a more generous learning scenario is assumed in which the learner is in addition given oracle access to the target distribution $\mathbf{Z}$-i.e., it can submit an input $x$ and get back the probability mass that $\mathbf{Z}$ assigns to $x$.)

More recently, Freund and Mansour [14] gave an efficient algorithm for learning a mixture of two general product distributions over $\{0,1\}^{n}$. Very roughly speaking, their algorithm uses a "hold-out" set of attributes to approximately reconstruct the line passing through the two means $\mathbf{E}\left[\mathbf{X}^{1}\right], \mathbf{E}\left[\mathbf{X}^{2}\right]$ of the product distributions $\mathbf{X}^{1}$ and $\mathbf{X}^{2}$; the algorithm then performs a one-dimensional search on this line for the optimal pair of centers to maximize the likelihood of the data. Around the same time Cryan [8] and Cryan, Goldberg, and Goldberg [9] gave an efficient algorithm for learning phylogenetic trees in the two-state general Markov model. Their algorithm has several stages including estimating covariances between different pairs of leaves, partitioning the leaves into "related sets," constructing a tree for each related set, and then generating an overall tree topology. For the special case in which the tree topology is a star, this gives an algorithm for learning an arbitrary mixture of two product distributions over $\{0,1\}^{n}$. Both [14] and [8] stated as an open question the problem of obtaining a polynomial-time algorithm for learning a mixture of $k>2$ product distributions. Indeed, recent work of Mossel and Roch [20] on learning phylogenetic trees argues that the rank-deficiency of transition matrices is a major source of difficulty, and this may indicate why $k=2$ has historically been a barrier - a two-row matrix can be rank-deficient only if one row is a multiple of the other, whereas the general case of $k>2$ is much more complex.

In other related work, there is a vast literature in statistics on the general problem of analyzing mixture data-see $[19,21,23]$ for surveys. To a large degree this

\footnotetext{
${ }^{1}$ The KL divergence is often defined in terms of $\log _{2}$. It is more convenient for us to use ln, and it is easy to see that this choice does not affect our results. We remind the reader (see, e.g., [7]) that $\left\|\mathbf{Z}-\mathbf{Z}^{\prime}\right\|_{1} \leq \sqrt{2} \sqrt{\operatorname{KL}\left(\mathbf{Z} \| \mathbf{Z}^{\prime}\right)}$, where $\|\cdot\|_{1}$ denotes total variation distance; hence if the KL divergence is small, then the total variation distance is also small.

${ }^{2}$ Of course, the algorithm works for product distributions over $\Sigma^{n}$ for any alphabet $\Sigma$ with $|\Sigma|=b$; i.e., the names of the characters in the alphabet do not matter.
} 
work centers on trying to find the exact best mixture model (in terms of likelihood) which explains a given data sample; this is computationally intractable in general. In contrast, our main goal (and the goal of $[18,14,9,8,20]$ ) is to obtain efficient algorithms that produce $\epsilon$-close hypotheses.

We also note that there has been recent interest in learning mixtures of $n$ dimensional Gaussians from the point of view of clustering $[10,11,2,24]$. In this framework one is given samples from a mixture of "well-separated" Gaussians, and the goal is to classify each point in the sample according to which Gaussian it came from. We discuss the relationship between our scenario and this recent literature on Gaussians in section 11; here we emphasize that throughout this paper we make no "separation" assumptions (indeed, no assumptions at all) on the component product distributions in the mixture.

Finally, the problem of learning discrete mixture distributions may have applications to other areas of theoretical computer science, such as database privacy $[22,6]$ and quantum complexity [1].

1.3. Our results. In this paper we give an efficient algorithm for learning a mixture of $k=O(1)$ many product distributions over $\{0,1\}^{n}$. Our main theorem is the following.

THEOREM 1. Fix any $k=O(1)$, and let $\mathbf{Z}$ be any unknown mixture of $k$ product distributions over $\{0,1\}^{n}$. Then there is an algorithm that, given samples from $\mathbf{Z}$ and any $\epsilon, \delta>0$ as inputs, runs in time $\operatorname{poly}(n / \epsilon) \cdot \log (1 / \delta)$ and with probability $1-\delta$ outputs a mixture $\mathbf{Z}^{\prime}$ of $k$ product distributions over $\{0,1\}^{n}$ satisfying $\operatorname{KL}\left(\mathbf{Z} \| \mathbf{Z}^{\prime}\right) \leq \epsilon$.

We emphasize that our algorithm requires none of the additional assumptionssuch as minimum mixing weights or coordinate means being bounded away from 0 , $1 / 2$, or 1 - that appear in some work on learning mixture distributions.

Our algorithm runs in time $(n / \epsilon)^{O\left(k^{3}\right)}$, which is polynomial only if $k$ is constant; however, this dependence may be unavoidable. In Theorem 18 we give a reduction from a difficult open question in computational learning theory (the problem of learning decision trees of superconstant size) to the problem of learning a mixture of any superconstant number of product distributions over $\{0,1\}^{n}$. This implies that solving the mixture learning problem for any $k=\omega(1)$ would require a major breakthrough in learning theory, and suggests that the dependence on $k$ in the exponent of the running time may be unavoidable.

We also generalize our result to learn a mixture of product distributions over $\{0, \ldots, b-1\}^{n}$ for any constant $b$, as follows.

TheOrEm 2. Fix any $k=O(1)$ and $b=O(1)$, and let $\mathbf{Z}$ be any unknown mixture of $k$ product distributions over $\{0, \ldots, b-1\}^{n}$. Then there is an algorithm that, given samples from $\mathbf{Z}$ and any $\epsilon, \delta>0$ as inputs, runs in time $\operatorname{poly}(n / \epsilon) \cdot \log (1 / \delta)$ and with probability $1-\delta$ outputs a mixture $\mathbf{Z}^{\prime}$ of $k$ product distributions over $\{0, \ldots, b-1\}^{n}$ satisfying $\mathrm{KL}\left(\mathbf{Z} \| \mathbf{Z}^{\prime}\right) \leq \epsilon$.

Taking $b=k$, this gives a polynomial-time algorithm for learning $k$-state Markov evolutionary trees (METs) with a star topology. (Note that the main result of $[9,8]$ is an algorithm for learning two-state METs with an arbitrary topology; hence our result is not comparable to theirs.)

\section{Overview of our approach.}

2.1. The WAM algorithm. The cornerstone of our overall learning algorithms is an algorithm we call WAM (for weights and means). WAM is an algorithm that takes as input a parameter $\epsilon>0$ and has access to samples from an unknown mixture 
$\mathbf{Z}$ of $k$ product distributions $\mathbf{X}^{1}, \ldots, \mathbf{X}^{k}$. Here each $\mathbf{X}^{i}=\left(\mathbf{X}_{1}^{i}, \ldots, \mathbf{X}_{n}^{i}\right)$ is an $\mathbf{R}^{n}$ valued random vector with independent coordinates. The goal of WAM is to output accurate estimates for all of the mixing weights $\pi^{i}$ and coordinate means $\mu_{j}^{i}:=\mathbf{E}\left[\mathbf{X}_{j}^{i}\right]$. Note that a product distribution over $\{0,1\}^{n}$ is completely specified by its coordinate means.

More precisely, WAM outputs a list of $\operatorname{poly}(n / \epsilon)$ many candidates $\left(\left\langle\hat{\pi}^{1}, \ldots, \hat{\pi}^{k}\right\rangle\right.$, $\left.\left\langle\hat{\mu}_{1}^{1}, \hat{\mu}_{2}^{1}, \ldots, \hat{\mu}_{n}^{k}\right\rangle\right)$; each candidate may be viewed as a possible estimate for the correct mixing weights and coordinate means. We will show that with high probability at least one of the candidates output by WAM is parametrically accurate; roughly speaking, this means that the candidate is a good estimate in the sense that $\left|\hat{\pi}^{i}-\pi^{i}\right| \leq \epsilon$ for each $i$ and that $\left|\hat{\mu}_{j}^{i}-\mu_{j}^{i}\right| \leq \epsilon$ for each $i$ and $j$. However, there is a slight twist: if a mixing weight $\pi^{i}$ is very low, then WAM may not receive any samples from $\mathbf{X}^{i}$, and thus it is not reasonable to require that WAM get an accurate estimate for $\mu_{1}^{i}, \ldots, \mu_{n}^{i}$. On the other hand, if $\pi^{i}$ is so low, then it is not very important to get an accurate estimate for $\mu_{1}^{i}, \ldots, \mu_{n}^{i}$ because $\mathbf{X}^{i}$ has only a tiny effect on $\mathbf{Z}$. We thus make the following formal definition.

Definition 3. A candidate $\left(\left\langle\hat{\pi}^{1}, \ldots, \hat{\pi}^{k}\right\rangle,\left\langle\hat{\mu}_{1}^{1}, \hat{\mu}_{2}^{1}, \ldots, \hat{\mu}_{n}^{k}\right\rangle\right)$ is said to be parametrically $\epsilon$-accurate if

1. $\left|\hat{\pi}^{i}-\pi^{i}\right| \leq \epsilon$ for all $1 \leq i \leq k$;

2. $\left|\hat{\mu}_{j}^{i}-\mu_{j}^{i}\right| \leq \epsilon$ for all $1 \leq i \leq k$ and $1 \leq j \leq n$ such that $\pi^{i} \geq \epsilon$.

The main technical theorem in this paper, Theorem 4 , shows that so long as the $\mathbf{X}^{i}$ 's take values in a bounded range, WAM will with high probability output at least one candidate that is parametrically accurate. The proof of this theorem uses tools from linear algebra (singular value theory) along with a very careful error analysis.

Remark 1. As will be clear from the proof of Theorem 4, WAM will succeed even if the mixture distributions $\mathbf{X}^{i}$ are only pairwise independent, not fully independent. This may be of independent interest.

2.2. From WAM to PAC learning (binary case). As we noted already, in the binary case a product distribution on $\{0,1\}^{n}$ is completely specified by its $n$ coordinate means; thus a candidate can essentially be viewed as a hypothesis mixture of product distributions. (This is not precisely correct, as the candidate mixing weights may not precisely sum to 1 and the candidate means might be outside the range $[0,1]$ by as much as $\epsilon$.) To complete the learning algorithm described in Theorem 1 we must give an efficient procedure that takes the list output by WAM and identifies a candidate distribution that is close to $\mathbf{Z}$ in KL divergence, as required by Theorem 1 . We do this in two steps:

1. We first give an efficient procedure that converts a parametrically accurate candidate into a proper hypothesis distribution that is close to $\mathbf{Z}$ in $\mathrm{KL}$ divergence. We apply this procedure to each candidate in the list output by WAM, and thus obtain a list of mixtures (hypotheses), at least one of which is close to $\mathbf{Z}$ in $\mathrm{KL}$ divergence.

2. We then show that a maximum-likelihood procedure can take a list of hypotheses, at least one of which is good (close to $\mathbf{Z}$ in KL divergence), and identify a single hypothesis which is good.

2.3. Larger alphabets. In the larger alphabet setting, $\mathbf{Z}$ is a mixture of $k$ product distributions $\mathbf{X}^{1}, \ldots, \mathbf{X}^{k}$ over $\{0, \ldots, b-1\}^{n}$. Now each mixture component $\mathbf{X}^{i}$ is defined by $b n$ parameters $p_{j, \ell}^{i}($ with $j=1, \ldots, n$ and $\ell=0, \ldots, b-1)$, where $p_{j, \ell}^{i}$ is the probability that a draw from $\mathbf{X}_{j}^{i}$ yields $\ell$. The simple but useful observation 
that underlies our extension to $\{0, \ldots, b-1\}^{n}$ is the following: just as any distribution over $\{0,1\}$ is completely specified by its mean, any distribution $\mathbf{X}_{j}^{i}$ over $\{0, \ldots, b-1\}$ is completely specified by its first $b-1$ moments $\mathbf{E}\left[\mathbf{X}_{j}^{i}\right], \mathbf{E}\left[\left(\mathbf{X}_{j}^{i}\right)^{2}\right], \ldots, \mathbf{E}\left[\left(\mathbf{X}_{j}^{i}\right)^{b-1}\right] .^{3}$ Our approach is thus to run WAM $b-1$ times; for $\ell=1, \ldots, b-1$ the $\ell$ th run will sample from the given mixture distribution and convert each sample $\left(z_{1}, \ldots, z_{n}\right)$ to the sample $\left(z_{1}^{\ell}, \ldots, z_{n}^{\ell}\right)$. We then carefully combine the lists output by the runs of WAM, and follow steps similar to 1 and 2 above to find a good hypothesis in the combined list.

2.4. Outline. Section 3 is dedicated to explaining the ideas behind the WAM algorithm and its proof of correctness. The detailed algorithm and proof are then presented in section 4 . We discuss the application of WAM to the $b$-ary case in section 5. The two steps outlined in section 2.2 are conceptually straightforward, but the details are quite technical; they are given in sections 6 through 8 . The pieces are all put together to prove Theorem 2 in section 9 (note that Theorem 1 is a special case of Theorem 2).

In section 10 we detail our reduction from a difficult open question in computational learning theory. We conclude in section 11 with a discussion of applications and future work.

3. The WAM algorithm. In this section we describe our main algorithm, WAM. We assume a general mixture setting: WAM has access to samples from $\mathbf{Z}$, a mixture of $k$ product distributions $\mathbf{X}^{1}, \ldots, \mathbf{X}^{k}$ with mixing weights $\pi^{1}, \ldots, \pi^{k}$. Each $\mathbf{X}^{i}=\left(\mathbf{X}_{1}^{i}, \ldots, \mathbf{X}_{n}^{i}\right)$ is an $n$-dimensional random variable. We will further assume that all components' coordinates are bounded in the range $[-1,1]$; i.e., $\mathbf{X}^{i} \in[-1,1]^{n}$ with probability 1 . We have chosen $[-1,1]$ for mathematical convenience; by scaling and translating samples we can get a theorem about any interval such as $[0,1]$ or $\left[0,(b-1)^{b-1}\right]$, with an appropriate scaling of $\epsilon$. We write $\mu_{j}^{i}:=\mathbf{E}\left[\mathbf{X}_{j}^{i}\right] \in[-1,1]$ for the mean of the $j$ th coordinate of $\mathbf{X}^{i}$.

Our main theorem is the following.

THEOREM 4. There is an algorithm WAM with the following property: for any $k=O(1)$ and any $\epsilon, \delta>0$, WAM runs in time $\operatorname{poly}(n / \epsilon) \cdot \log (1 / \delta)$ and outputs a list of $\operatorname{poly}(n / \epsilon)$ many candidates, at least one of which (with probability at least $1-\delta$ ) is parametrically $\epsilon$-accurate.

We give the full proof of correctness in section 4.2. The remainder of this section is devoted to explaining the main ideas behind the algorithm and its analysis.

3.1. Overview of WAM. There is of course a brute-force way to come up with a list of candidates $\left(\left\langle\hat{\pi}^{1}, \ldots, \hat{\pi}^{k}\right\rangle,\left\langle\hat{\mu}_{1}^{1}, \hat{\mu}_{2}^{1}, \ldots, \hat{\mu}_{n}^{k}\right\rangle\right)$, at least one of which is parametrically $\epsilon$-accurate: simply "try all possible values" for the parameters up to additive accuracy $\epsilon$. In other words, try all values $0, \epsilon, 2 \epsilon, 3 \epsilon, \ldots, 1$ for the mixing weights and all values $-1,-1+\epsilon, \ldots, 1-\epsilon, 1$ for the means. We call this approach "gridding." Unfortunately there are $\Theta(n)$ parameters in a candidate, so this naive gridding strategy requires time (and produces a list of length) $(1 / \epsilon)^{\Theta(n)}$, i.e., exponential in $n$, which is clearly unacceptable.

The basic idea behind WAM is as follows: given all pairwise correlations between the coordinates of $\mathbf{Z}$, it can be shown that there are a constant number of "key" parameters that suffice to determine all others. Hence in polynomial time we can

\footnotetext{
${ }^{3}$ This is the case since the distribution can be recovered from the moments by solving a system of linear equations based on a Vandermonde matrix, which has full rank.
} 
empirically estimate all the correlations, try all possibilities for the constantly many key parameters, and then determine the remaining $\Theta(n)$ parameters.

The main challenge in implementing this idea is that it is not at all a priori clear that the error incurred from gridding the key parameters does not "blow up" when these are used to determine the remaining parameters. The heart of our analysis involves showing that it suffices to grid the key parameters to granularity poly $(\epsilon / n)$ in order to get final error $\epsilon$.

3.2. The algorithm, and intuition for the analysis. We will now go over the steps of the algorithm WAM and at the same time provide an "intuitive" discussion of the analysis. A concise description of the steps of WAM is given at the start of section 4 for the reader's convenience. Throughout this section we will assume for the sake of discussion that the steps we take incur no error; a sketch of the actual error analysis appears in section 3.3.

The first step of WAM is to "grid" the values of the mixing weights $\left\{\pi^{i}\right\}$ to granularity $\epsilon_{\mathrm{wts}}:=\epsilon^{3}$. Since there are only constantly many mixing weights, this costs just a multiplicative factor of poly $(1 / \epsilon)$ in the running time. The remainder of the algorithm then assumes that the mixing weights are known. These mixing weights are of course approximate, but for the purposes of this intuitive description of WAM, we will simply assume that we have exactly correct values for $\left\{\pi^{i}\right\}$.

The next step is simple: suppose that some $s$ of the $k$ mixing weights we have are smaller than $\epsilon$. By the definition of being " $\epsilon$-parametrically accurate," we are not obliged to worry about coordinates with such small mixing weights; hence we will simply forget about these mixture components completely and treat $k$ as $k-s$ in what follows. (We assign arbitrary values for the candidate means of the forgotten components.) We may henceforth assume that $\pi^{i} \geq \epsilon>0$ for all $i$.

The next step of algorithm WAM is to use samples from $\mathbf{Z}$ to estimate the pairwise correlations between the coordinates of $\mathbf{Z}$. Specifically, for all pairs of coordinates $1 \leq j<j^{\prime} \leq n$, the algorithm WAM empirically estimates

$$
\operatorname{corr}\left(j, j^{\prime}\right)=\mathbf{E}\left[\mathbf{Z}_{j} \mathbf{Z}_{j^{\prime}}\right]
$$

The estimation will be done to within additive accuracy $\epsilon_{\text {matrix }}=\operatorname{poly}(\epsilon / n)$; specifically, $\epsilon_{\text {matrix }}:=\tau^{k+1}$, where $\tau:=\epsilon^{2} / n^{2}$. With high (i.e., $1-\delta$ ) confidence we will get good such estimates in time $\operatorname{poly}(n / \epsilon) \cdot \log (1 / \delta)$. Again, for the purposes of this intuitive description of WAM we will henceforth assume that we have exactly correct values for each value $\operatorname{corr}\left(j, j^{\prime}\right)$. (As an aside, this is the only part of the algorithm that uses samples from $\mathbf{Z}$; as we will shortly see, this justifies Remark 1.)

Observe that since $\mathbf{X}_{j}^{i}$ and $\mathbf{X}_{j^{\prime}}^{i}$ are (pairwise) independent we have

$$
\operatorname{corr}\left(j, j^{\prime}\right)=\mathbf{E}\left[\mathbf{Z}_{j} \mathbf{Z}_{j^{\prime}}\right]=\sum_{i=1}^{k} \pi^{i} \mathbf{E}\left[\mathbf{X}_{j}^{i} \mathbf{X}_{j^{\prime}}^{i}\right]=\sum_{i=1}^{k} \pi^{i} \mathbf{E}\left[\mathbf{X}_{j}^{i}\right] \mathbf{E}\left[\mathbf{X}_{j^{\prime}}^{i}\right]=\sum_{i=1}^{k} \pi^{i} \mu_{j}^{i} \mu_{j^{\prime}}^{i}
$$

Let us define

$$
\tilde{\mu}_{j}^{i}=\sqrt{\pi^{i}} \mu_{j}^{i}
$$

and write $\tilde{\mu}_{j}=\left(\tilde{\mu}_{j}^{1}, \tilde{\mu}_{j}^{2}, \ldots, \tilde{\mu}_{j}^{k}\right) \in[-1,1]^{k}$ for $1 \leq j \leq n$. We thus have

$$
\operatorname{corr}\left(j, j^{\prime}\right)=\tilde{\mu}_{j} \cdot \tilde{\mu}_{j^{\prime}},
$$




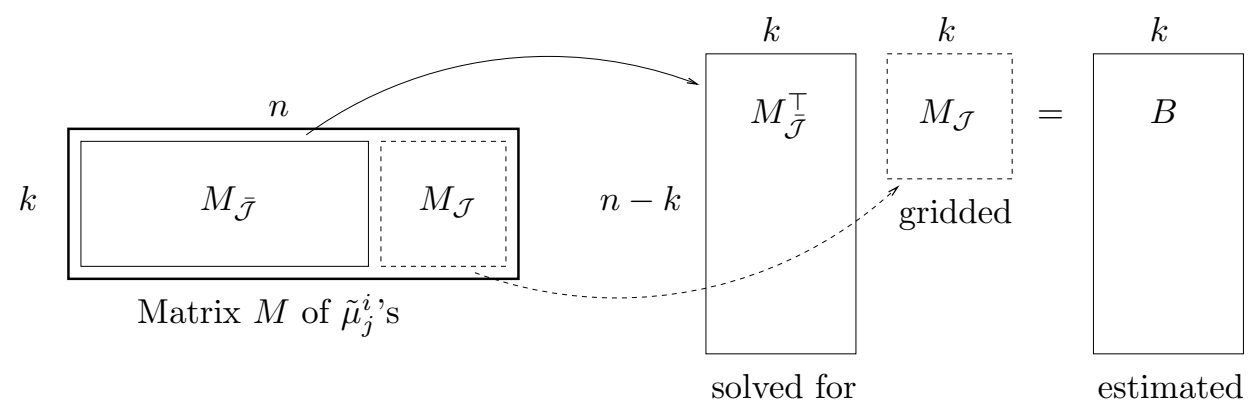

FIG. 1. The full rank case. We solve for the unknown $\tilde{\mu}_{j}^{i}$ 's in $M_{\overline{\mathcal{J}}}$ using the gridded values in $M_{\mathcal{J}}$ and the values in $B$ estimated directly from the samples.

where denotes the dot product in $\mathbf{R}^{k}$. The remaining task for WAM is to determine all the values $\mu_{j}^{i}$. Since WAM already has values for each $\pi^{i}$ and each $\pi^{i} \geq \epsilon>0$, it suffices for WAM to determine all the values $\tilde{\mu}_{j}^{i}$ and then divide by $\sqrt{\pi^{i}}$.

At this point WAM has empirically estimated values for all the pairwise dot products $\tilde{\mu}_{j} \cdot \tilde{\mu}_{j^{\prime}}, j \neq j^{\prime}$, and as mentioned, for intuitive purposes we are assuming that all of these estimates are exactly correct. Let $M$ denote the $k \times n$ matrix whose $(i, j)$ entry is the unknown $\tilde{\mu}_{j}^{i}$; i.e., the $j$ th column of $M$ is $\tilde{\mu}_{j}$. The statement that WAM has all the $\operatorname{dot}$ products $\tilde{\mu}_{j} \cdot \tilde{\mu}_{j^{\prime}}$ for $j \neq j^{\prime}$ is equivalent to saying that WAM has all the off-diagonal entries of the Gram matrix $M^{\top} M$. We are thus led to what is essentially the central problem WAM solves:

Central task. Given (estimates) for the off-diagonal entries of the $n \times n$ Gram matrix $M^{\top} M$, generate (estimates of) all possible candidates for the entries of the $k \times n$ matrix $M$

(Note: The diagonal entries of $M^{\top} M$ are the quantities $\tilde{\mu}_{j} \cdot \tilde{\mu}_{j}=\sum_{i=1}^{k} \pi^{i}\left(\mu_{j}^{i}\right)^{2}$, and there is no obvious way to estimate these quantities using samples from $\mathbf{Z}$. Also there are $n$ such quantities, which is too many to "grid over." Nevertheless, the fact that we are missing the diagonal entries of $M^{\top} M$ will not play an important role for WAM.)

In general, a complete $n \times n$ Gram matrix determines the original $k \times n$ matrix up to isometries on $\mathbf{R}^{k}$. Such isometries can be described by $k \times k$ orthonormal matrices, and these $k^{2}$ "degrees of freedom" roughly correspond to the constantly many key parameters that we grid over in the end. A geometric intuition for the central task is the following: there are $n$ unknown vectors in $\mathbf{R}^{k}$ and we have all the "angles" (more precisely, the dot products) between them. Thus fixing $k$ of the vectors (hence $k^{2}$ unknown coordinates) is enough to completely determine the remainder of the vectors.

The full rank case. We proceed with our intuitive description of WAM and show how to solve the central task when $M$ has full rank. Having done this, we will give the actual steps of the algorithm that show how the full rank assumption can be removed.

So suppose for now that $M$ has full rank. Then there exists some set of $k$ columns of $M$ that are linearly independent, say $\mathcal{J}=\left\{j_{1}, \ldots, j_{k}\right\} \subset[n]$. Algorithm WAM tries all $\left(\begin{array}{l}n \\ k\end{array}\right)=\operatorname{poly}(n)$ possibilities for the set $\mathcal{J}$ and then grids over the vectors $\tilde{\mu}_{j_{1}}, \ldots, \tilde{\mu}_{j_{k}}$ with granularity $\epsilon_{\text {matrix }}=\operatorname{poly}(\epsilon / n)$ in each coordinate. As usual for the purposes of intuition, we assume that we now have $\tilde{\mu}_{j_{1}}, \ldots, \tilde{\mu}_{j_{k}}$ exactly correct.

Let $M_{\mathcal{J}}$ be the $k \times k$ matrix given by the $\mathcal{J}$-columns of $M$, and let $M_{\overline{\mathcal{J}}}$ be the $k \times(n-k)$ matrix given by deleting the $\mathcal{J}$-columns of $M$. WAM now has the entries 
of $M_{\mathcal{J}}$ and must compute the remaining unknowns, $M_{\overline{\mathcal{J}}}$. Since WAM has all of the off-diagonal entries of $M^{\top} M$, it has all of the values of $B=M_{\mathcal{J}}^{\top} M_{\mathcal{J}}$. (See Figure 1.) However, the columns of $M_{\mathcal{J}}$ are linearly independent, so $M_{\mathcal{J}}$ is invertible, and hence WAM can compute $M_{\mathcal{J}}^{\top}=B M_{\mathcal{J}}^{-1}$ in poly $(n)$ time. Having done this, WAM has all the entries of $M$, and so the central task is complete, as is the algorithm.

The general case. Of course, in general, $M$ does not have full rank. This represents the main conceptual problem we faced in rigorously solving the central task. Indeed, we believe that handling rank-deficiency is the chief conceptual problem for the whole learning mixtures question, and that our linear algebraic methods for overcoming it (the description of which occupies the remainder of section 3) are the main technical contribution of this paper.

Suppose $\operatorname{rank}(M)=r<k$. By trying all possible values (only constantly many), algorithm WAM can be assumed to know $r$. Now by definition of $\operatorname{rank}(M)=r$ there must exist $k-r$ orthonormal vectors $u_{r+1}, \ldots, u_{k} \in[-1,1]^{k}$ which are orthogonal to all columns of $M$. WAM grids over these vectors with granularity $\epsilon_{\text {matrix }}$, incurring another multiplicative poly $(n / \epsilon)$ factor in the running time. As usual, assume for the intuitive discussion that we now have the $u_{j}$ 's exactly. Let these vectors be adjoined as columns to $M$, forming $M^{\prime}$. But now the matrix $M^{\prime}$ has full rank; furthermore, WAM knows all the off-diagonal elements of $\left(M^{\prime}\right)^{\top} M^{\prime}$, i.e., all the pairwise dot products of $M^{\prime}$ 's columns, since all of the new dot products which involve the $u_{j}$ 's are simply 0 ! Thus we now have an instance of the central task with a full rank matrix, a case we already solved. (Technically, $n$ may now be as large as $n+(k-1)$, but this is still $O(n)$, and hence no time bound is affected.) Solving the central task on $M^{\prime}$ (which contains all the entries of $M$ ) completes the algorithm WAM in the rank-deficient case.

3.3. Sketch of the actual analysis of WAM. The preceding intuitive discussion of algorithm WAM neglected all error analysis. Correctly handling the error analysis is the somewhat subtle issue we discuss in this section. As mentioned, the full proof is given in section 4.2.

The main issue in the error analysis comes in understanding the right notion of the rank of $M$ - since all of our gridding inevitably yields only approximations of the entries of $M$, the actual notion of rank is far too fragile to be of use. Recall the outline of the algorithm in our idealized intuition (rank-deficient case):

$$
\begin{aligned}
& r=\text { dimension of subspace in which } \tilde{\mu}_{j} \text { 's lie } \\
& \quad \Rightarrow \text { augment } M \text { by } k-r \text { orthogonal } u_{i} \text { 's, forming } M^{\prime} \Rightarrow M^{\prime} \text { now full rank } \\
& \quad \Rightarrow \text { find nonsingular } k \times k \text { submatrix } M_{\mathcal{J}}^{\prime} \Rightarrow \text { solve linear system } M_{\overline{\mathcal{J}}}^{\prime} M_{\mathcal{J}}^{\prime}=B .
\end{aligned}
$$

For the purposes of the error analysis, we reinterpret the operation of WAM as follows:

$$
\begin{aligned}
& \text { (1) } r^{*}=\text { dimension of subspace in which the } \tilde{\mu}_{j} \text { 's "essentially" lie } \\
& \Rightarrow \text { augment } M \text { by } k-r \text { "essentially" orthogonal } u_{i} \text { 's, forming } M^{\prime} \\
& \qquad M^{\prime} \text { now "strongly" full rank }
\end{aligned}
$$

The real difficulty of the error analysis comes in the last step: controlling the error incurred from solving the linear system. Since we will have only approximately correct values for the entries of $M_{\mathcal{J}}^{\prime}$ and $B$, we need to analyze the additive error arising from 
solving a perturbed linear system. Standard results from numerical analysis (see Proposition 9 in section 4.1) allow us to bound this error by a function of (i) the error in $M_{\mathcal{J}}^{\prime}$ and $B$, and (ii) the smallest singular value of $M_{\mathcal{J}}^{\prime}$, denoted by $\sigma_{k}\left(M^{\prime}\right)$. More precisely, as we describe in Proposition 9, the error is bounded by the errors in $M_{\mathcal{J}}$ and $B$ normalized by $\sigma_{k}\left(M^{\prime}\right)$.

Let us briefly recall some notions related to singular values. Given any $k \times n$ matrix $M$, the first (largest) singular value of $M$ is $\sigma_{1}(M)=\max _{\left\|u_{1}\right\|_{2}=1}\left\|u_{1}^{\top} M\right\|_{2}$, and a $u_{1}$ achieving this maximum is taken as the first (left) singular vector of $M$. The second singular value of $M$ is $\sigma_{2}(M)=\max _{\left\|u_{2}\right\|_{2}=1, u_{2} \perp u_{1}}\left\|u_{2}^{\top} M\right\|_{2}$, and $u_{2}$ is the second left singular vector of $M$. In general, the $i$ th singular value and vector are given by maximizing over all $\left\|u_{i}\right\|_{2}=1$ orthogonal to all $u_{1}, \ldots, u_{i-1}$. In a welldefined sense (the Frobenius norm), the smallest singular value $\sigma_{k}(M)$ measures the distance of $M$ from being singular.

WAM's final error bounds arise from dividing the error in its estimates for $M_{\mathcal{J}}^{\prime}$ and $B$ by the smallest singular value of $M_{\mathcal{J}}^{\prime}$. The error in the estimates for the entries of $M_{\mathcal{J}}^{\prime}$ come from gridding, and thus can essentially be made as small as desired; WAM makes them smaller than $\epsilon_{\text {matrix }}$. The errors in $B$ come from two sources: some of the entries of $B$ are estimates of quantities $\tilde{\mu}_{j} \cdot \tilde{\mu}_{j^{\prime}}=\operatorname{corr}\left(j, j^{\prime}\right)$, and again these errors can be made essentially as small as desired, smaller than $\epsilon_{\text {matrix }}$. However, the other errors in $B$ come from approximating the quantities $\tilde{\mu}_{j} \cdot u_{i}$ by 0 , i.e., assuming that the augmenting vectors are orthogonal to the columns of $M$.

As the reader may by now have guessed, the vectors with which WAM attempts to augment $M$ will be the last $k-r^{*}$ singular vectors of $M, u_{r^{*}+1}, \ldots, u_{k}$. The hope is that for an appropriate choice of $r^{*}$ these singular vectors will be "essentially" orthogonal to the columns of $M$, and that the resulting $M^{\prime}$ will be "strongly" full rank, in the sense that $\sigma_{k}\left(M^{\prime}\right)$ will be somewhat large (cf. (1)). One can show (see Proposition 8 of section 4.1) that the extent to which the $u_{i}$ 's are orthogonal to the columns of $M$ is controlled by the $\left(r^{*}+1\right)$ th singular value of $M$; i.e., $\left|\tilde{\mu}_{j} \cdot u_{i}\right| \leq$ $\sigma_{r^{*}+1}(M)$ for all $i \geq r^{*}+1$; this is precisely the error we incur for the zero entries in $B$. On the other hand, one can also show that the augmented $M^{\prime}$ has smallest singular value at least $\sigma_{r^{*}}(M)$. Thus we are motivated to choose $r^{*}$ so as to get a large multiplicative gap between $\sigma_{r^{*}}(M)$ and $\sigma_{r^{*}+1}(M)$, as follows.

Definition 5. Given $\tau>0$, the $\tau$-essential rank of $M$ is

$$
r^{*}(M)=r_{\tau}^{*}(M)=\min \left\{0 \leq r \leq k: \sigma_{r+1}(M) / \sigma_{r}(M) \leq \tau\right\},
$$

where we take $\sigma_{0}(M)=1$ and $\sigma_{k+1}(M)=0$.

One might think that if the additive error incurred from solving the linear system were to be roughly $\sigma_{r^{*}}(M) / \sigma_{r^{*}+1}(M)$, then it should suffice to select $\tau$ on the order of poly $(\epsilon)$. However, there is still a missing piece of the analysis: although the smallest singular value of $M^{\prime}$ becomes at least $\sigma_{r^{*}}(M)$ after adjoining the $u_{j}$ 's, we use only a $k \times k$ submatrix $M_{\mathcal{J}}^{\prime}$ to solve the linear system. Is it the case that if $M^{\prime}$ has a large smallest singular value then its "best" $k \times k$ submatrix also has a somewhat large smallest singular value? We need a quantitative version of the fact that a nonsingular $k \times n$ matrix has a $k \times k$ nonsingular submatrix (again, cf. (1)).

This does not seem to be a well-studied problem, and indeed there are some open questions in linear algebra surrounding the issue. It is possible to derive an extremely weak quantitative result of the required nature using the Cauchy-Binet formula. We instead give the following quantitatively strong version.

Corollary 6. Let $A$ be a $k \times n$ real matrix with $\sigma_{k}(A) \geq \epsilon$. Then there exists a subset of columns $\mathcal{J} \subseteq[n]$ with $|\mathcal{J}|=k$ such that $\sigma_{k}\left(A_{\mathcal{J}}\right) \geq \epsilon / \sqrt{k(n-k)+1}$. 
(We call the result a corollary because our proof in section 4.1 is derived from a 1997 linear algebraic result of Goreinov, Tyrtyshnikov, and Zamarashkin [15]. Incidentally, it is conjectured in their paper that $\sqrt{k(n-k)+1}$ can be replaced by $\sqrt{n}$.)

With this result in hand it becomes sufficient to take $\tau=\epsilon^{2} / n^{2}$, as described in the previous section. Now the error analysis can be completed:

- If $M$ has a singular value gap of $\tau$ and so has essential rank $r^{*}<k$, then when WAM tries out the appropriate $r^{*}$ and singular vectors, the error it incurs from solving the linear system is roughly at most $O(\sqrt{n} \tau)=O\left(\epsilon^{2} / n^{3 / 2}\right)$, and as we show at the end of section 4.2 , having this level of control over errors in solving the linear system for the unknown $\tilde{\mu}_{j}^{i}$ 's lets us obtain the final $\mu_{j}^{i}$ values to the required $\epsilon$-accuracy.

- On the other hand, if $M$ has no singular value gap smaller than $\tau$, then its smallest singular value is at least $\tau^{k}$; thus it suffices to take $\epsilon_{\text {matrix }}=\tau^{k+1}=$ poly $(\epsilon / n)$ to control the errors in the full rank case.

See section 4.2 for the detailed proof of correctness.

4. Algorithm WAM. Algorithm WAM has access to samples from the mixture $\mathbf{Z}$ and takes as input parameters $\epsilon, \delta>0$.

Algorithm WAM.

1. Let $\epsilon_{\mathrm{wts}}=\epsilon^{3}, \tau=\epsilon^{2} / n^{2}$, and $\epsilon_{\text {matrix }}=\tau^{k+1}$.

2. Grid over the mixing weights, producing values $\hat{\pi}^{1}, \ldots, \hat{\pi}^{k} \in[0,1]$ accurate to within $\pm \epsilon_{\mathrm{wts}}$. If $s$ of these weights are smaller than $\epsilon-\epsilon_{\mathrm{wts}}$, eliminate them and treat $k$ as $k-s$ in what follows.

3. Make empirical estimates $\widehat{\operatorname{corr}}\left(j, j^{\prime}\right)$ for all correlations $\operatorname{corr}\left(j, j^{\prime}\right)=\mathbf{E}\left[\mathbf{Z}_{j} \mathbf{Z}_{j^{\prime}}\right]=\tilde{\mu}_{j} \cdot \tilde{\mu}_{j^{\prime}}$ for $j \neq j^{\prime}$ to within $\pm \epsilon_{\text {matrix }}$, with confidence $1-\delta$.

4. Let $M$ be the $k \times n$ matrix of unknowns $\left(M_{i j}\right)=\left(\tilde{\mu}_{j}^{i}\right)$, and try all possible integers $0 \leq r^{*} \leq k$ for the essential rank of $M$.

5. Grid over $k-r^{*}$ vectors $\hat{u}_{r^{*}+1}, \ldots, \hat{u}_{k} \in[-1,1]^{k}$ to within $\pm \epsilon_{\text {matrix }}$ in each coordinate and augment $M$ with these as columns, forming $\widehat{M}^{\prime}$.

6. Try all possible subsets of exactly $k$ column indices of $\widehat{M}^{\prime}$; write these indices as $\mathcal{J}=J \cup J^{\prime}$, where $J$ corresponds to columns from the original matrix $M$ and $J^{\prime}$ corresponds to augmented columns. Grid over $[-1,1]$ for the entries of $M$ in columns $J$ to within $\pm \epsilon_{\text {matrix }}$, yielding $\left\{\hat{\tilde{\mu}}_{j}^{i}: i \in[k], j \in J\right\}$. Let $\widehat{M}_{\mathcal{J}}^{\prime}$ denote the matrix of estimates for all the columns in $\mathcal{J}$. (See Figure 2.)

7. Let $\overline{\mathcal{J}}$ denote the columns of $M$ other than $J$, and let $M_{\overline{\mathcal{J}}}$ denote the matrix of remaining unknowns formed by these columns. Let $\widehat{B}$ be the matrix with rows indexed by $\overline{\mathcal{J}}$ and columns indexed by $\mathcal{J}$ whose $\left(j, j^{\prime}\right)$ entry is the estimate $\widehat{\operatorname{corr}}\left(j, j^{\prime}\right)$ of $\tilde{\mu}_{j} \cdot \tilde{\mu}_{j^{\prime}}$ if $j^{\prime} \in J$, or is 0 if $j^{\prime} \in J^{\prime}$. Using the entries of $\hat{B}$ and $\widehat{M}_{\mathcal{J}}^{\prime}$ (all of which are known), solve the system $M_{\mathcal{J}}^{\top} \widehat{M}_{\mathcal{J}}^{\prime}=\widehat{B}$ to obtain estimates $\hat{\tilde{\mu}}_{j}^{i}$ for the entries of $M_{\overline{\mathcal{J}}}$ (which are the unknown $\tilde{\mu}_{j}^{i}$ 's), thus producing estimates $\hat{\tilde{\mu}}_{j}^{i}$ for all entries of $M$. (If the matrix $\widehat{M}_{\mathcal{J}}^{\prime}$ is singular, simply abandon the current 


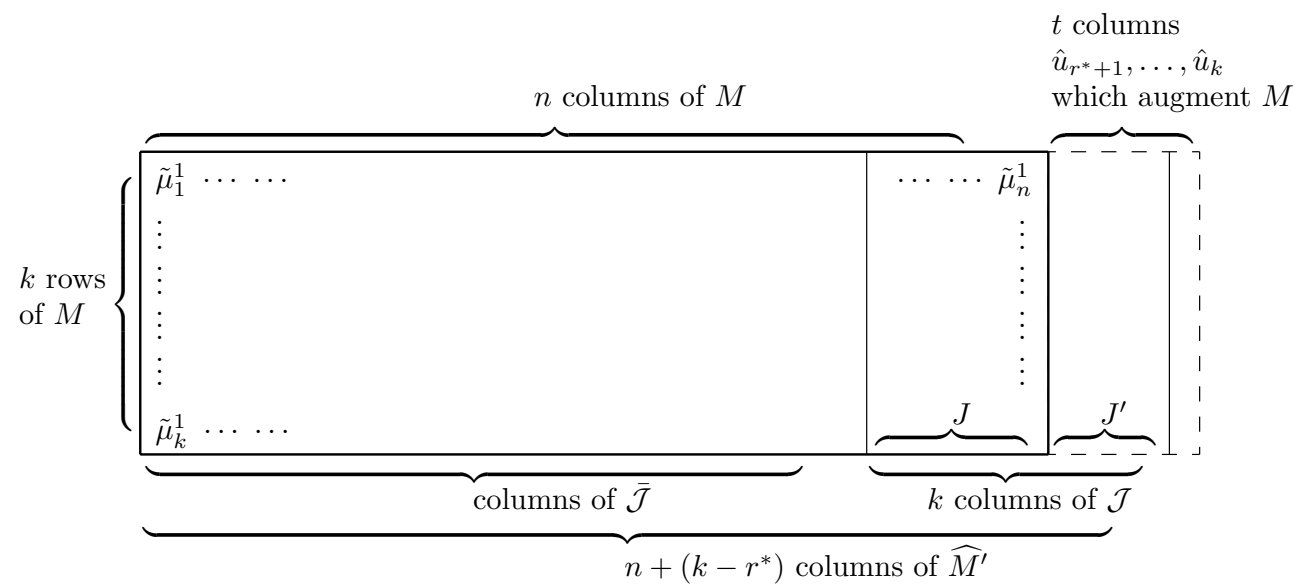

FIG. 2. A depiction of the matrix used by WAM. For ease of illustration the columns $J$ of M are depicted as being the rightmost columns of $M$, and the columns $J^{\prime}$ from the augmenting columns $\hat{u}_{k-t+1}, \ldots, \hat{u}_{k}$ are depicted as being the leftmost of those augmenting columns.

gridding. )

8. From the estimated values $\hat{\tilde{\mu}}_{j}^{i}$, compute the estimates $\hat{\mu}_{j}^{i}=\hat{\tilde{\mu}}_{j}^{i} / \sqrt{\hat{\pi}^{i}}$ for all $i, j$. (Note that $\hat{\pi}^{i}$ is never 0 , since each is at least $\left.\epsilon-\epsilon_{\mathrm{wts}}>0.\right)$

9. Output the candidate $\left(\left\langle\hat{\pi}^{1}, \ldots, \hat{\pi}^{k}\right\rangle,\left\langle\hat{\mu}_{1}^{1}, \hat{\mu}_{2}^{1}, \ldots, \hat{\mu}_{n}^{k}\right\rangle\right)$.

4.1. Linear algebra necessities. In this section we give the results from linear algebra and numerical analysis necessary for the analysis of WAM.

Let $A=\left(a_{i j}\right)$ be any $k \times n$ real matrix, and write its singular value decomposition as $A=U \Sigma V$. Here $U$ is a $k \times k$ matrix with orthonormal columns $u_{1}, \ldots, u_{k}, \Sigma$ is a $k \times k$ diagonal matrix with $\sigma_{1}(A) \geq \cdots \geq \sigma_{k}(A) \geq 0$ on the diagonal, and $V$ is a $k \times n$ matrix with orthonormal rows. We let $\sigma_{1}(A) \geq \cdots \geq \sigma_{k}(A) \geq 0$ denote the singular values of $A$, and let $u_{1}, \ldots, u_{k}$ denote the corresponding left singular vectors of $A$, i.e., the columns of $U$. If it is clear from context, we simply write $\sigma_{i}$ for $\sigma_{i}(A)$. Recall that

- the vectors $u_{1}, \ldots, u_{k}$ form an orthonormal basis for $\mathbf{R}^{k}$;

- $\sigma_{1}=\max _{\|x\|_{2}=1}\left\|x^{\top} A\right\|_{2}$ and $\sigma_{k}=\min _{\|x\|_{2}=1}\left\|x^{\top} A\right\|_{2}$.

The Frobenius norm $\|A\|_{F}$ of a $k \times n$ matrix $A$ is defined as $\|A\|_{F}=\sqrt{\sum_{i, j}\left(A_{i, j}\right)^{2}}$. We recall the well-known fact that $\sigma_{k}(A)$ equals the Frobenius norm distance from the $k \times n$ matrix $A$ to the nearest rank-deficient matrix $\tilde{A}$, i.e.,

$$
\sigma_{k}(A)=\min _{\operatorname{rank}(\tilde{A})<k}\|A-\tilde{A}\|_{F} .
$$

The spectral norm $\|A\|_{2}$ of a $k \times n$ matrix $A$ is $\|A\|_{2}=\max _{\|x\|_{2}=1}\|A x\|$. It is well known that $\|A\|_{2}=\sigma_{1}$ and $\|A\|_{F}=\sqrt{\sigma_{1}^{2}+\cdots+\sigma_{k}^{2}}$; note that this implies $\|A\|_{2} \leq\|A\|_{F}$.

Our first necessary result is a quantitative version of the elementary fact that a full rank $k \times n$ matrix has a full rank $k \times k$ submatrix. We will use the following theorem of Goreinov, Tyrtyshnikov, and Zamarashkin [15].

THEOREM 7 (see [15]). Let $V$ be a $k \times n$ real matrix with orthonormal rows. Then there is a $k \times k$ submatrix $V_{J}$ which has $\sigma_{k}\left(V_{J}\right) \geq 1 / \sqrt{k(n-k)+1}$. 
The result we need is an easy corollary, Corollary 6 given above.

Proof of Corollary 6. Recall that by the singular value decomposition we have $A=U \Sigma V$, where $U$ is a $k \times k$ matrix with orthonormal columns, $\Sigma$ is a $k \times k$ diagonal matrix with diagonal entries $\sigma_{1}, \ldots, \sigma_{k}$, and $V$ is a $k \times n$ matrix with orthonormal rows. Let $V_{J}$ be the $k \times k$ submatrix of $V$ whose existence is asserted by Theorem 7, so $\sigma_{k}\left(V_{J}\right) \geq 1 / \sqrt{k(n-k)+1}$. We have $\sigma_{k}(U)=1$ (since $U$ is an orthogonal matrix) and $\sigma_{k}(\Sigma) \geq \epsilon$, so

$$
\sigma_{k}\left(U \Sigma V_{J}\right) \geq \sigma_{k}(U) \sigma_{k}(\Sigma) \sigma_{k}\left(V_{J}\right) \geq \epsilon / \sqrt{k(n-k)+1}
$$

where the inequality holds since $\sigma_{k}(P Q) \geq \sigma_{k}(P) \sigma_{k}(Q)$ for any $k \times k$ matrices $P, Q$. (This is easily seen from the variational characterization $\sigma_{k}(P)=\min _{\|x\|_{2}=1}\left\|x^{\top} P\right\|_{2}$.) The corollary follows by observing that $U \Sigma V_{J}$ is the $k \times k$ submatrix of $A$ whose columns are in $J$.

The next result we will need is the characterization of what happens when the last $k-r^{*}$ left singular vectors of a matrix are adjoined to it.

Proposition 8. Let $A$ be $a k \times n$ matrix with columns $a_{1}, \ldots, a_{n}$. Fix any $r^{*}$, and let $u_{r^{*}+1}, \ldots, u_{k}$ be the left singular vectors corresponding to the smallest singular values $\sigma_{r^{*}+1}, \ldots, \sigma_{k}$ of $A$. Let $A^{\prime}$ be $A$ with the vectors $u_{r^{*}+1}, \ldots, u_{k}$ adjoined as columns. Then

$$
\sigma_{k}\left(A^{\prime}\right) \geq \min \left\{1, \sigma_{r^{*}}(A)\right\},
$$

and for all $r^{*}+1 \leq \ell \leq k$ and for all columns $a_{j}$ of $A$ we have

$$
\left|a_{j} \cdot u_{\ell}\right| \leq \sigma_{r^{*}+1}(A) .
$$

Proof. Write the singular value decomposition $A=U \Sigma V$, where $U$ is a $k \times k$ matrix with orthonormal columns $u_{1}, \ldots, u_{k}, \Sigma$ is a $k \times k$ diagonal matrix with $\sigma_{1} \geq$ $\cdots \geq \sigma_{k} \geq 0$ on the diagonal, and $V$ is a $k \times n$ matrix with orthonormal rows. It follows that for any vector $x \in \mathbf{R}^{k}$ we have

$$
\left\|x^{\top} A\right\|_{2}^{2}=\sigma_{1}^{2}\left(x^{\top} u_{1}\right)^{2}+\cdots+\sigma_{k}^{2}\left(x^{\top} u_{k}\right)^{2} .
$$

Let $R$ denote the $k \times\left(k-r^{*}\right)$ matrix whose columns are $u_{r^{*}+1}, \ldots, u_{k}$, so we have $A^{\prime}=$ $\left[\begin{array}{ll}A R & R\end{array}\right.$. It is easily verified that the left singular vectors of $R$ are simply $u_{r^{*}+1}, \ldots, u_{k}$, while the singular values of $R$ are all 1 . Consequently we have

$$
\left\|x^{\top} R\right\|_{2}^{2}=\left(x^{\top} u_{r^{*}+1}\right)^{2}+\cdots+\left(x^{\top} u_{k}\right)^{2}
$$

for any $x \in \mathbf{R}^{k}$.

Now recall the variational characterization of $\sigma_{k}\left(A^{\prime}\right)$, namely $\sigma_{k}\left(A^{\prime}\right)=$ $\min _{\|x\|_{2}=1}\left\|x^{\top} A^{\prime}\right\|_{2}$. Since $\left\|x^{\top} A^{\prime}\right\|_{2}=\sqrt{\left\|x^{\top} A\right\|_{2}^{2}+\left\|x^{\top} R\right\|_{2}^{2}}$, we have

$$
\sigma_{k}\left(A^{\prime}\right)=\min _{\|x\|_{2}=1} \sqrt{\sigma_{1}^{2}\left(x^{\top} u_{1}\right)^{2}+\cdots+\sigma_{k}^{2}\left(x^{\top} u_{k}\right)^{2}+\left(x^{\top} u_{r^{*}+1}\right)^{2}+\cdots+\left(x^{\top} u_{k}\right)^{2}} .
$$

Since $u_{1}, \ldots, u_{k}$ form an orthonormal basis for $\mathbf{R}^{k}$ we have that $\left(x^{\top} u_{1}\right)^{2}+\cdots+$ $\left(x^{\top} u_{k}\right)^{2}=1$ for all $\|x\|_{2}=1$. If we let $\alpha_{x}=\left(x^{\top} u_{r^{*}+1}\right)^{2}+\cdots+\left(x^{\top} u_{k}\right)^{2}$, then the quantity inside the square root of $(2)$ is at least $\sigma_{r^{*}}^{2}\left(1-\alpha_{x}\right)+\alpha_{x} \geq \min \left\{\sigma_{r^{*}}^{2}, 1\right\}$. This proves the first inequality of the proposition. 
For the second inequality, we observe that $a_{j} \cdot u_{\ell}=u_{\ell}^{\top} U \Sigma v_{j}$, where $v_{j}$ is the $j$ th column of $V$. Since $U$ is orthonormal and $\Sigma_{\ell, \ell}=\sigma_{\ell}$ we thus have

$$
\left|u_{\ell}^{\top} U \Sigma v_{j}\right|=\left|\sigma_{\ell} v_{\ell, j}\right| \leq \sigma_{\ell} \leq \sigma_{r^{*}+1},
$$

where the first inequality holds since the rows of $V$ are orthonormal, and hence each entry of $V$ must be at most 1 in magnitude.

The final result we will need involves controlling the error in a perturbed linear system in terms of the smallest singular value. Although we could not find the following statement in the literature, it should be considered a very basic result from numerical analysis.

Proposition 9. Let $A$ be a nonsingular $k \times k$ matrix, $b$ be a $k$-dimensional vector, and $x$ be the solution to $A x=b$. Assume that $\|x\|_{\infty} \leq 1$. Suppose $A^{\prime}$ is a $k \times k$ matrix such that each entry of $A-A^{\prime}$ is at most $\epsilon_{\text {matrix }}$ in magnitude, and assume that $\epsilon_{\text {matrix }}<\sigma_{k}(A) / 2 k$. Let $b^{\prime}$ be a $k$-dimensional vector satisfying $\left\|b-b^{\prime}\right\|_{\infty} \leq \epsilon_{\mathrm{rhs}}$. Let $x^{\prime}$ be the solution to $A^{\prime} x^{\prime}=b^{\prime}$. Then we have

$$
\left\|x-x^{\prime}\right\|_{\infty} \leq O\left(k^{3 / 2}\right) \frac{\epsilon_{\text {matrix }}+\epsilon_{\text {rhs }}}{\sigma_{k}(A)} .
$$

Proof. Write $E=A-A^{\prime}$ and $\eta=b-b^{\prime}$. By our assumption on $A^{\prime}$ we have $\|E\|_{F}=\left\|A-A^{\prime}\right\|_{F} \leq k \epsilon_{\text {matrix }}$. By our assumption on $\epsilon_{\text {matrix }}$ this is at most $\sigma_{k}(A) / 2$. It follows that $\sigma_{k}\left(A^{\prime}\right) \geq \sigma_{k}(A) / 2$, and in particular $A^{\prime}$ is nonsingular. Thus $x^{\prime}$ is indeed well defined, and we may write

$$
\begin{aligned}
A x-A x^{\prime}=E x^{\prime}+\eta & \Rightarrow x-x^{\prime} \\
\Rightarrow\left\|x-x^{\prime}\right\|_{2} & \leq \| A^{-1}\left(E x^{\prime}+\eta\right) \\
& \leq \frac{1}{\sigma_{k}(A)}\left(\left(k \epsilon_{\text {matrix }}\right)\left(\|x-\|_{2}\|\|_{2}+\|x\|_{2}\right)+\left(\sqrt{k} \epsilon_{\text {rhs }}\right)\right) \\
\Rightarrow\left(\sigma_{k}(A)-k \epsilon_{\text {matrix }}\right)\left\|x-x^{\prime}\right\|_{2} & \leq k \epsilon_{\text {matrix }}\|x\|_{2}+\sqrt{k} \epsilon_{\text {rhs }} .
\end{aligned}
$$

We now use $\sigma_{k}(A)-k \epsilon_{\text {matrix }} \geq \sigma_{k}(A) / 2$ to conclude

$$
\left\|x-x^{\prime}\right\|_{2} \leq \frac{2\left(k \epsilon_{\text {matrix }}\|x\|_{2}+\sqrt{k} \epsilon_{\mathrm{rhs}}\right)}{\sigma_{k}(A)} .
$$

Finally, $\left\|x-x^{\prime}\right\|_{\infty} \leq\left\|x-x^{\prime}\right\|_{2}$ and $\|x\|_{2} \leq \sqrt{k}$ complete the proof.

4.2. Proof of Theorem 4. We go through the algorithm step by step, as it appears at the start of section 4. In step 1 of WAM, we define constants $\epsilon_{\mathrm{wts}}=\epsilon^{3}$, $\tau=\epsilon^{2} / n^{2}$, and $\epsilon_{\text {matrix }}=\tau^{k+1}$, which we use throughout the proof.

In step 2 of WAM, the algorithm will grid over estimates $\hat{\pi}^{i}$ that satisfy $\left|\hat{\pi}^{i}-\pi^{i}\right| \leq$ $\epsilon_{\mathrm{wts}}$ for all $i$. In this case, any mixing component $\mathbf{X}^{i}$ whose mixing weight $\pi^{i}$ is at least $\epsilon$ will not be eliminated. Since we need not be concerned with accuracy for the means of the other mixing components, we can ignore them and assume for the rest of the proof that $\pi^{i} \geq \epsilon$ for all $i$.

Now we come to the main work in the proof of correctness of Theorem 4: namely, showing that in steps 3-7 of Algorithm WAM, accurate estimates for the $\tilde{\mu}_{j}^{i}$ 's are produced. Our goal for most of the remainder of the proof will be to show that we obtain estimates $\hat{\tilde{\mu}}_{j}^{i}$ satisfying

$$
\left|\hat{\tilde{\mu}}_{j}^{i}-\tilde{\mu}_{j}^{i}\right| \leq \tilde{\epsilon}:=\epsilon^{2}
$$

Copyright $@$ by SIAM. Unauthorized reproduction of this article is prohibited. 
for all $i$.

To that end, let $r^{*}=r_{\tau}^{*}(M)$, the $\tau$-essential rank of $M$. We will quickly dismiss the two easy cases, $r^{*}=0$ and $r^{*}=k$; we then treat the general case $0<r^{*}<k$.

$r^{*}=0$ case. By definition, in this case $\sigma_{1}(M) \leq \tau \leq \tilde{\epsilon}$. Since $\sigma_{1}(M)$ is at least as large as the magnitude of $M$ 's largest entry, we must therefore have $\left|\tilde{\mu}_{j}^{i}\right| \leq \tilde{\epsilon}$ for all $i, j$. Now when WAM tries $r^{*}=0$ in step 4 , tries the $k$ standard basis vectors for $\hat{u}_{1}, \ldots, \hat{u}_{k}$ in step 5 , and chooses all of these vectors for $\mathcal{J}$ in step 6 , it will set $\widehat{B}=0$ in step 7 and get $\hat{\tilde{\mu}}_{j}^{i}=0$ for all $i, j$ when it solves the linear system. But this is indeed within an additive $\tau \leq \tilde{\epsilon}$ of the true values, as desired.

$r^{*}=k$ case. By definition, it's not hard to see that in this case we must have $\sigma_{k}(M) \geq \tau^{k}$. Now consider when WAM tries $r^{*}=k$ in step 4 . Step 5 becomes vacuous. By Corollary 6 there is some set of $k$ columns $\mathcal{J}=J$ such that $\sigma_{k}\left(M_{\mathcal{J}}\right) \geq$ $\sigma_{k}(M) / \sqrt{k(n-k)+1} \geq \tau^{k} / n$. In step 6 , WAM will try out this $\mathcal{J}$ and grid the associated entries to within $\pm \epsilon_{\text {matrix. }}$. In step 7 the algorithm will use only $\widehat{\text { corr }}$ 's in forming $\widehat{B}$, and these will also be correct to within an additive $\pm \epsilon_{\text {matrix }}$. We can now use Proposition 9-note that $\epsilon_{\text {matrix }}=\tau^{k+1} \leq\left(\tau^{k} / n\right) / 2 k \leq \sigma_{k}\left(M_{\mathcal{J}}\right) / 2 k$, as necessary. This gives estimates in step 7 satisfying

$$
\left|\hat{\tilde{\mu}}_{j}^{i}-\tilde{\mu}_{j}^{i}\right| \leq O\left(k^{3 / 2}\right) \frac{2 \epsilon_{\text {matrix }}}{\tau^{k} / n}=O\left(k^{3 / 2} n \tau\right) \leq \tilde{\epsilon},
$$

as desired.

$0<r^{*}<k$ case. In this case, by definition of the essential rank, we have

$$
\tau \sigma_{r^{*}}(M) \geq \sigma_{r^{*}+1}(M) \geq \tau^{k} .
$$

In step 4 WAM will try out the correct value for $r^{*}$, and in step 5 WAM will grid over vectors $\hat{u}_{r^{*}+1}, \ldots, \hat{u}_{k}$ that are within $\pm \epsilon_{\text {matrix }}$ in each coordinate of the actual last left singular vectors of $M, u_{r^{*}+1}, \ldots, u_{k}$. Let $M^{\prime}$ denote the matrix $M$ with these true singular vectors adjoined. By Proposition 8 we have

$$
\sigma_{k}\left(M^{\prime}\right) \geq \min \left\{1, \sigma_{r^{*}}(M)\right\}
$$

From the crude upper bound $\sigma_{r^{*}}(M) \leq\|M\|_{F}=\sqrt{\sum_{i, j}\left(\tilde{\mu}_{j}^{i}\right)^{2}} \leq \sqrt{k n}$, we can restate (4) as simply $\sigma_{k}\left(M^{\prime}\right) \geq \sigma_{r^{*}}(M) / \sqrt{k n}$. Now applying Corollary 6 , we conclude there is a subset $\mathcal{J}$ of $M^{\prime}$ 's columns with $|\mathcal{J}|=k$ such that

$$
\sigma_{k}\left(M_{\mathcal{J}}^{\prime}\right) \geq \sigma_{k}\left(M^{\prime}\right) / \sqrt{k(n-k)+1} \geq \sigma_{r^{*}}(M) / k n .
$$

In step 6 , WAM will try this set of columns $\mathcal{J}=J \cup J^{\prime}$; it will also grid estimates for the entries in this column that are correct up to an additive $\pm \epsilon_{\text {matrix }}$. Note that WAM now has an $\widehat{M_{\mathcal{J}}^{\prime}}$ that has all entries correct up to an additive $\pm \epsilon_{\text {matrix }}$. Now consider the matrix $\widehat{B}$ WAM forms in step 7 . For the columns corresponding to $J$ the entries are given by $\widehat{\text { corr's, }}$, which are correct to within $\pm \epsilon_{\text {matrix }}$. For the columns corresponding to $J^{\prime}$ the entries are 0's; by the second part of Proposition 8 these are correct up to an additive $\sigma_{r^{*}+1}(M)$. We now use Corollary 6 to bound the error resulting from solving the system $M_{\mathcal{\mathcal { J }}}^{\top} \widehat{M}_{\mathcal{J}}^{\prime}=\widehat{B}$ in step 7 . To check that the necessary hypothesis is satisfied we combine (3) and (5):

$$
\sigma_{k}\left(M_{\mathcal{J}}^{\prime}\right) / 2 k \geq \sigma_{r^{*}}(M) / 2 k^{2} n \geq \tau^{k-1} / 2 k^{2} n \geq \tau^{k+1}=\epsilon_{\text {matrix }}
$$


Now Proposition 9 tells us that the $\hat{\tilde{\mu}}_{j}^{i}$ produced satisfy

$\left|\hat{\tilde{\mu}}_{j}^{i}-\tilde{\mu}_{j}^{i}\right| \leq O\left(k^{3 / 2}\right) \frac{\epsilon_{\text {matrix }}+\max \left\{\epsilon_{\text {matrix }}, \sigma_{r^{*}+1}(M)\right\}}{\sigma_{k}\left(M_{\mathcal{J}}^{\prime}\right)} \leq O\left(k^{5 / 2} n\right) \frac{\epsilon_{\text {matrix }}+\sigma_{r^{*}+1}(M)}{\sigma_{r^{*}}(M)}$,

where in the last step we used (5). But by (3) we have $\epsilon_{\text {matrix }} / \sigma_{r^{*}}(M) \leq \epsilon_{\text {matrix }} / \tau^{k-1}=$ $\tau^{2}$ and also $\sigma_{r^{*}+1}(M) / \sigma_{r^{*}}(M) \leq \tau$. Thus we have

$$
\left|\hat{\tilde{\mu}}_{j}^{i}-\tilde{\mu}_{j}^{i}\right| \leq O\left(k^{5 / 2} n\right) \tau \leq \tilde{\epsilon}
$$

as desired.

It remains to bound the error blowup in step 8. By this point we have values for the $\pi^{i}$ 's that are accurate to within $\pm \epsilon_{\mathrm{wts}}$, and further, all $\pi^{i}$ 's are at least $\epsilon$. We also have values for all $\tilde{\mu}_{j}^{i}$,s that are accurate to within $\pm \tilde{\epsilon}$. Since the function $g(x, y)=y / \sqrt{x}$ satisfies

$$
\sup _{\substack{x \in[\epsilon, 1] \\ y \in[-1,1]}}\left|\frac{\partial}{\partial x} g(x, y)\right|=\frac{1}{2} \epsilon^{-3 / 2} \text { and } \sup _{\substack{x \in[\epsilon, 1] \\ y \in[-1,1]}}\left|\frac{\partial}{\partial y} g(x, y)\right|=\epsilon^{-1 / 2},
$$

the mean value theorem implies that in step 8 our resulting estimates $\hat{\mu}_{j}^{i}$ are accurate to within additive error

$$
\epsilon_{\mathrm{wts}} \cdot \frac{1}{2} \epsilon^{-3 / 2}+\tilde{\epsilon} \cdot \epsilon^{-1 / 2} \leq \epsilon
$$

as necessary.

This completes the proof of WAM's correctness. As for the running time, it is easy to see that the dominating factor comes from gridding over the entries of $M_{J}$ and $u_{r^{*}+1}, \ldots, u_{k}$. Since there are $k^{2}$ entries and we grid to granularity $\epsilon_{\text {matrix }}=\tau^{k+1}=$ $\operatorname{poly}(n / \epsilon)^{k}$, the overall running time is $\operatorname{poly}(n / \epsilon)^{k^{3}}$; i.e., $\operatorname{poly}(n / \epsilon)$ for constant $k$.

5. Estimating higher moments. In this section we explain our remarks from section 2.3 more thoroughly; specifically, how to use WAM to learn a mixture $\mathbf{Z}$ of $k$ product distributions $\mathbf{X}^{1}, \ldots, \mathbf{X}^{k}$ over $\{0, \ldots, b-1\}^{n}$. Such a distribution can be "parametrically" described by mixing weights $\left\{\pi^{i}\right\}_{i \in[k]}$ and probabilities $\left\{p_{j, \ell}^{i}\right\}$, where $p_{j, \ell}^{i}=\operatorname{Pr}\left[\mathbf{X}_{j}^{i}=\ell\right]$.

Running WAM on samples from $\mathbf{Z}$ gives a list of estimates of mixing weights and coordinate means $\mathbf{E}\left[\mathbf{X}_{j}^{i}\right]$, but these coordinate means are insufficient to completely describe the distributions $\mathbf{X}_{j}^{i}$. However, suppose that we run WAM on samples from $\mathbf{Z}^{\ell}$ (i.e., each time we obtain a draw $\left(z_{1}, \ldots, z_{n}\right)$ from $\mathbf{Z}$, we actually give $\left(z_{1}^{\ell}, \ldots, z_{n}^{\ell}\right)$ to WAM). It is easy to see that, by doing this, we are running WAM on the $\pi$ weighted mixture of distributions $\left(\mathbf{X}^{1}\right)^{\ell}, \ldots,\left(\mathbf{X}^{k}\right)^{\ell}$; we will thus get as output a list of candidates for the mixing weights and the coordinate $\ell$ th moments $\mathbf{E}\left[\left(\mathbf{X}_{j}^{i}\right)^{\ell}\right]$ for $\mathbf{Z}$.

Our algorithm for distributions over $\{0, \ldots, b-1\}^{n}$ uses this approach to obtain a list of candidate descriptions of each of the first $b-1$ coordinate moments of $\mathbf{Z}$. The algorithm then essentially takes the cross-product of these $b-1$ lists to obtain a list of overall candidates, each of which is an estimate of the mixing weights and all $b-1$ moments. Since WAM guarantees that each list contains an accurate estimate, the overall list will also contain an accurate estimate of the mixing weights and of all moments. For each candidate the estimate of the moments is then easily converted to "parametric form" $\left\{p_{j, \ell}^{i}\right\}$, and as we show, any candidate with accurate estimates of the moments yields an accurate estimate of the probabilities $p_{j, \ell}^{i}$.

Copyright (c) by SIAM. Unauthorized reproduction of this article is prohibited. 
We now give the main theorem of the section, the proof of which contains the details of the algorithm.

TheOREM 10. Fix $k=O(1), b=O(1)$. Let $\mathbf{Z}$ be a mixture of $k$ product distributions $\mathbf{X}^{1}, \ldots, \mathbf{X}^{k}$ over $\{0, \ldots, b-1\}^{n}$, so $\mathbf{Z}$ is described by mixing weights $\pi^{1}, \ldots, \pi^{k}$ and probabilities $\left\{p_{j, \ell}^{i}\right\}_{i \in[k], j \in[n], \ell \in\{0, \ldots, b-1\}}$.

There is an algorithm with the following property: for any $\epsilon, \delta>0$, the algorithm runs in poly $(n / \epsilon) \cdot \log \frac{1}{\delta}$ time, and with probability $1-\delta$ outputs a list of candidates $\left\langle\left\{\hat{\pi}^{i}\right\},\left\{\hat{p}_{j, \ell}^{i}\right\}\right\rangle$ such that for at least one candidate in the list, the following hold:

1. $\left|\hat{\pi}^{i}-\pi^{i}\right| \leq \epsilon$ for all $i \in[k]$; and

2. $\left|\hat{p}_{j, \ell}^{i}-p_{j, \ell}^{i}\right| \leq \epsilon$ for all $i, j, \ell$ such that $\pi^{i} \geq \epsilon$.

Proof. For each $\ell=1, \ldots, b-1$, the algorithm runs WAM on the random variable $\mathbf{Z}^{\ell}$. In each such run, the " $\epsilon$ " parameter of WAM is set to $\epsilon^{\prime}:=\epsilon \sigma_{b} /\left(O\left(b^{3 / 2}\right)\right.$. $\left.(b-1)^{b-1}\right)$, where $\sigma_{b}$ is a constant we define later, and the " $\delta$ " parameter is set to $\delta^{\prime}:=\delta /(b-1)$. From these runs we obtain $(b-1)$ lists $L_{1}, \ldots, L_{b-1}$ of candidates $\left\langle\left\{\hat{\pi}^{i}\right\},\left\{\hat{\mu}_{j, \ell}^{i}\right\}_{i, j}\right\rangle$, where $\hat{\mu}_{j, \ell}^{i}$ is an estimate of $\mu_{j, \ell}^{i}=\mathbf{E}\left[\left(X_{j}^{i}\right)^{\ell}\right]$. The algorithm then uses these $(b-1)$ lists to construct one larger list $L$ of candidates $\left\langle\left\{\hat{\pi}^{i}\right\},\left\{\mu_{j, \ell}^{i}\right\}_{i, j, \ell}\right\rangle$, where each candidate estimates the mixing weights and all $b-1$ moments. This is done by taking all possible combinations of one candidate from each of the $b-1$ lists $L_{1}, \ldots, L_{b-1}$ and combining them as follows: take the mixing weights $\left\{\hat{\pi}^{i}\right\}$ from the candidate from list $L_{1}$, and for $\ell=1, \ldots, b-1$, take $\left\{\mu_{j, \ell}^{i}\right\}_{i, j}$ from the candidate from list $L_{\ell}$. The list $L$ will have size $|L|=\prod_{\ell=1}^{b-1}\left|L_{\ell}\right|=\operatorname{poly}(n, 1 / \epsilon)$.

Theorem 4 on the WAM algorithm guarantees that with probability at least $1-(b-1) \delta^{\prime}=1-\delta$, each list $L_{\ell}$ contains a candidate whose $\left\{\hat{\mu}_{j, \ell}^{i}\right\}$ are accurate estimates of the $\ell$ th moments. When we choose the accurate candidate from each list, we will obtain an overall candidate in $L$ that is accurate on all $b-1$ moments. Define $\epsilon^{\prime \prime}:=\epsilon^{\prime}(b-1)^{b-1} / 2=\epsilon \sigma_{b} / O\left(b^{3 / 2}\right)$. Formally, the list $L$ will contain a candidate $\left\langle\left\{\hat{\pi}^{i}\right\},\left\{\hat{\mu}_{j, \ell}^{i}\right\}_{i, j, \ell}\right\rangle$ such that (i) $\left|\hat{\pi}^{i}-\pi^{i}\right| \leq \epsilon^{\prime \prime}$ for all $i \in[k]$, and (ii) $\left|\hat{\mu}_{j, \ell}^{i}-\mu_{j, \ell}^{i}\right| \leq \epsilon^{\prime \prime}$ for all $i, j, \ell$ such that $\pi^{i} \geq \epsilon^{\prime \prime}$. (The extra factor of $(b-1)^{b-1} / 2$ comes from the need to scale the distributions for WAM so that the means fall into the range $[-1,1]$.)

To complete the proof of the theorem, we must show how the algorithm converts each candidate $\left\langle\left\{\hat{\pi}^{i}\right\},\left\{\hat{\mu}_{j, \ell}^{i}\right\}\right\rangle$ in the list $L$ into "parametric" form $\left\langle\left\{\hat{\pi}^{i}\right\},\left\{\hat{p}_{j, \ell}^{i}\right\}\right\rangle$ so that the "good" candidate satisfying (i) and (ii) above does not incur much error. It is easy to see that for a given $i \in[k], j \in[n]$, we have $\left(\mu_{j, 0}^{i}, \ldots, \mu_{j, b-1}^{i}\right)=\left(p_{j, 0}^{i}, \ldots, p_{j, b-1}^{i}\right) V$, where $V$ is a $b \times b$ Vandermonde matrix (more precisely, $V_{\alpha, \beta}=(\alpha-1)^{\beta-1}$, with $\left.V_{1,1}=1\right)$. Following this characterization, the algorithm computes $\left(\hat{p}_{j, 0}^{i}, \ldots, \hat{p}_{j, b-1}^{i}\right)=$ $\left(\hat{\mu}_{j, 0}^{i}, \ldots, \hat{\mu}_{j, b-1}^{i}\right) V^{-1}$ for each $i, j$ to obtain parametric estimates $\left\{\hat{p}_{j, \ell}^{i}\right\}$ for the probabilities $\left\{p_{j, \ell}^{i}\right\}$.

Now applying Proposition 9, we have that for all $i, j, \ell$ we have $\left|\hat{p}_{j, \ell}^{i}-p_{j, \ell}^{i}\right| \leq$ $\epsilon^{\prime \prime} \cdot O\left(b^{3 / 2}\right) / \sigma_{b}=\epsilon$, where $\sigma_{b}$ is set equal to $\sigma_{b}(V)$, the smallest singular value of $V$. (Since the Vandermonde matrix is nonsingular, even without specifying $\sigma_{b}$ we have that it is a positive constant that depends only on $b$; it can be shown to be at least $b^{- \text {poly }(b)}$.) The running time is dominated by the time to take the cross-product of the lists. This concludes the proof of Theorem 10.

We remark that the running time dependence on $b$ is of the form $(n / \epsilon)^{\text {poly }(b)}$; since $a b$ in the exponent is inevitable in our cross-product approach, we have refrained from excessive optimization of the dependence on $b$ (by doing things such as representing the alphabet by $b$ th roots of unity rather than equally spaced reals, which would have given a better Vandermonde singular value bound). 
6. The road ahead. Since the binary domain $\{0,1\}^{n}$ corresponds to the $b=2$ case of the general $\{0, \ldots, b-1\}^{n}$ domain, here we shall deal only with the latter.

Recall that $p_{j, \ell}^{i}$ is the probability that under the $i$ th product distribution over $\{0, \ldots, b-1\}^{n}$ in the target mixture $\mathbf{Z}$, the $j$ th coordinate takes value $\ell$. From Theorem 10, we have a list $L$ of $M$ candidates $\left\langle\left\{\hat{\pi}^{i}\right\},\left\{\hat{p}_{j, \ell}^{i}\right\}\right\rangle$ such that at least one candidate is parametrically accurate - i.e., satisfies the following:

1. $\left|\hat{\pi}^{i}-\pi^{i}\right| \leq \epsilon$ for all $i=1 \ldots k$; and

2. $\left|\hat{p}_{j, \ell}^{i}-p_{j, \ell}^{i}\right| \leq \epsilon$ for all $i \in[k], j \in[n]$ and $\ell \in\{0, \ldots, b-1\}$ such that $\pi^{i} \geq \epsilon$.

In section 7 , we show how to convert a candidate into a true mixture of product distributions, in such a way that any parametrically accurate candidate becomes a mixture distribution with small KL divergence from the target distribution (see Theorem 11). Applying this conversion procedure to the list from Theorem 10, we get a list of $M$ hypothesis mixture distributions such that at least one hypothesis in the list has small KL divergence from the target $\mathbf{Z}$ (see Theorem 15).

Then in section 8 we show how a maximum likelihood procedure can find a KLaccurate hypothesis (one with small KL divergence from $\mathbf{Z}$ ) from among a list of hypotheses, one of which is guaranteed to have good KL divergence (see Theorem 16).

In section 9 we combine Theorem 16 with Theorem 15 to obtain Theorem 2.

7. From candidates to hypothesis mixture distributions. The following theorem defines a process that converts a single candidate for the $\pi^{i}$ 's and $p_{j, \ell}^{i}$ 's of $\mathbf{Z}$ to a true mixture of product distributions over $\{0, \ldots, b-1\}^{n}$ that has at least some minimum mass on every point in $\{0, \ldots, b-1\}^{n}$. (As we will see in section 8 , this minimum mass condition is required by the maximum likelihood procedure.) More importantly, the theorem guarantees that if the candidate is parametrically accurate, then the process outputs a mixture distribution with small KL divergence relative to $\mathbf{Z}$.

THEOREM 11.

1. There is an efficient procedure $\mathcal{A}$ which takes values $\epsilon_{\mathrm{bprobs}}, \epsilon_{\mathrm{wts}}>0$ and $\hat{\pi}^{i}, \hat{p}_{j, \ell}^{i}$ as inputs and outputs a mixture $\dot{\mathbf{Z}}$ of $k$ product distributions over $\{0, \ldots, b-1\}^{n}$ with mixing weights $\dot{\pi}^{i}>0$ and probabilities $\dot{p}_{j, \ell}^{i}>0$ satisfying (a) $\sum_{i=1}^{k} \dot{\pi}^{i}=1$, and for each $i \in[k]$ and $j \in[n], \sum_{\ell=0}^{b-1} p_{j, \ell}^{i}=1$;

(b) $\dot{\mathbf{Z}}(x) \geq\left(\epsilon_{\text {bprobs }}\right)^{n}$ for all $x \in\{0, \ldots, b-1\}^{n}$.

2. Furthermore, suppose that $\mathbf{Z}$ is a mixture of $k$ product distributions on $\{0, \ldots, b-1\}^{n}$ with mixing weights $\pi^{1}, \ldots, \pi^{k}$ and probabilities $p_{j, \ell}^{i}$, and that the following are satisfied:

(a) for $i=1, \ldots, k$ we have $\left|\pi^{i}-\hat{\pi}^{i}\right| \leq \epsilon_{\mathrm{wts}}$, and

(b) for all $i, j, \ell$ such that $\pi^{i} \geq \epsilon_{\text {minwt }}$ we have $\left|p_{j, \ell}^{i}-\hat{p}_{j, \ell}^{i}\right| \leq \epsilon_{\text {bprobs }}$.

Then for sufficiently small $\epsilon_{\mathrm{bprobs}}$ and $\epsilon_{\mathrm{wts}}$ the mixture $\dot{\mathbf{Z}}$ will satisfy

$$
\mathrm{KL}(\mathbf{Z}|| \dot{\mathbf{Z}}) \leq \eta\left(\epsilon_{\mathrm{bprobs}}, \epsilon_{\mathrm{wts}}, \epsilon_{\mathrm{minwt}}\right),
$$

where

$$
\eta\left(\epsilon_{\mathrm{bprobs}}, \epsilon_{\mathrm{wts}}, \epsilon_{\text {minwt }}\right):=n \cdot\left(12 b^{3} \epsilon_{\mathrm{bprobs}}^{1 / 2}\right)+k \epsilon_{\text {minwt }} n \ln \left(b / \epsilon_{\mathrm{bprobs}}\right)+\epsilon_{\mathrm{wts}}^{1 / 3} .
$$

We prove Theorem 11 in section 7.2 after setting up the required machinery in section 7.1.

Copyright $@$ by SIAM. Unauthorized reproduction of this article is prohibited. 
7.1. Some tools. Here we give some propositions which will be used in the proof of Theorem 11.

The following simple proposition bounds the KL divergence between two product distributions in terms of the KL divergences between their coordinates.

Proposition 12. Suppose that $\mathbf{P}_{1}, \ldots, \mathbf{P}_{n}$ and $\mathbf{Q}_{1}, \ldots, \mathbf{Q}_{n}$ are distributions satisfying $\mathrm{KL}\left(\mathbf{P}_{i} \| \mathbf{Q}_{i}\right) \leq \epsilon_{i}$ for all $i$. Then $\mathrm{KL}\left(\mathbf{P}_{1} \times \cdots \times \mathbf{P}_{n} \| \mathbf{Q}_{1} \times \cdots \times \mathbf{Q}_{n}\right) \leq \sum_{i=1}^{n} \epsilon_{i}$.

Proof. We prove the case $n=2$ :

$$
\begin{aligned}
\mathrm{KL}\left(\mathbf{P}_{1} \times \mathbf{P}_{2} \| \mathbf{Q}_{1} \times \mathbf{Q}_{2}\right) & =\sum_{x} \sum_{y} \mathbf{P}_{1}(x) \mathbf{P}_{2}(y) \ln \frac{\mathbf{P}_{1}(x) \mathbf{P}_{2}(y)}{\mathbf{Q}_{1}(x) \mathbf{Q}_{2}(y)} \\
& =\sum_{x} \sum_{y} \mathbf{P}_{1}(x) \mathbf{P}_{2}(y) \ln \frac{\mathbf{P}_{1}(x)}{\mathbf{Q}_{1}(x)}+\sum_{x} \sum_{y} \mathbf{P}_{1}(x) \mathbf{P}_{2}(y) \ln \frac{\mathbf{P}_{2}(y)}{\mathbf{Q}_{2}(y)} \\
& =\sum_{y} \mathbf{P}_{2}(y) \operatorname{KL}\left(\mathbf{P}_{1} \| \mathbf{Q}_{1}\right)+\sum_{x} \mathbf{P}_{1}(x) \operatorname{KL}\left(\mathbf{P}_{2} \| \mathbf{Q}_{2}\right) \\
& \leq \epsilon_{1}+\epsilon_{2} .
\end{aligned}
$$

The general case follows by induction.

Very roughly speaking, the following proposition states that if $\mathbf{P}$ is a $\pi$-weighted mixture of distributions $\mathbf{P}^{1}, \ldots, \mathbf{P}^{k}$ and $\mathbf{Q}$ is a $\gamma$-weighted mixture of distributions $\mathbf{Q}^{1}, \ldots, \mathbf{Q}^{k}$, then if each $\mathbf{Q}^{i}$ is "close" to the corresponding $\mathbf{P}^{i}$ and the $\pi$-weighting is "close" to the $\gamma$-weighting, then $\mathbf{Q}$ is "close" to $\mathbf{P}$. To make this precise we need several technical conditions as stated in the proposition.

Proposition 13. Let $\pi^{1}, \ldots, \pi^{k}, \gamma^{1}, \ldots, \gamma^{k} \geq 0$ be mixing weights satisfying $\sum \pi^{i}=\sum \gamma^{i}=1$. Let $\epsilon_{1}, \epsilon_{2}, \epsilon_{3}, \epsilon_{\mathcal{I}}, \epsilon_{\text {all }}$ be positive constants. Let $\mathcal{I}=\left\{i: \pi^{i} \geq \epsilon_{3}\right\}$. Let $\mathbf{P}^{1}, \ldots, \mathbf{P}^{k}$ and $\mathbf{Q}^{1}, \ldots, \mathbf{Q}^{k}$ be distributions. Suppose that

1. $\left|\pi^{i}-\gamma^{i}\right| \leq \epsilon_{1}$ for all $i \in[k]$;

2. $\gamma^{i} \geq \epsilon_{2}$ for all $i \in[k]$;

3. $\mathrm{KL}\left(\mathbf{P}^{i} \| \mathbf{Q}^{i}\right) \leq \epsilon_{\mathcal{I}}$ for all $i \in \mathcal{I}$;

4. $\mathrm{KL}\left(\mathbf{P}^{i} \| \mathbf{Q}^{i}\right) \leq \epsilon_{\text {all }}$ for all $i \in[k]$.

Then, letting $\mathbf{P}$ denote the $\pi$-mixture of the $\mathbf{P}^{i}$ 's and $\mathbf{Q}$ the $\gamma$-mixture of the $\mathbf{Q}^{i}$ 's, for any $\epsilon_{4}>\epsilon_{1}$ we have

$$
\mathrm{KL}(\mathbf{P} \| \mathbf{Q}) \leq \epsilon_{\mathcal{I}}+k \epsilon_{3} \epsilon_{\mathrm{all}}+k \epsilon_{4} \ln \frac{\epsilon_{4}}{\epsilon_{2}}+\frac{\epsilon_{1}}{\epsilon_{4}-\epsilon_{1}} .
$$

Proof.

$$
\begin{aligned}
\mathrm{KL}(\mathbf{P} \| \mathbf{Q}) & =\sum_{x}\left(\sum_{i} \pi^{i} \mathbf{P}^{i}(x)\right) \ln \frac{\sum_{i} \pi^{i} \mathbf{P}^{i}(x)}{\sum_{i} \gamma^{i} \mathbf{Q}^{i}(x)} \\
& \leq \sum_{x} \sum_{i} \pi^{i} \mathbf{P}^{i}(x) \ln \frac{\pi^{i} \mathbf{P}^{i}(x)}{\gamma^{i} \mathbf{Q}^{i}(x)} \quad \text { (by the log-sum inequality [7]) } \\
& =\sum_{i} \pi^{i} \sum_{x}\left(\mathbf{P}^{i}(x) \ln \frac{\mathbf{P}^{i}(x)}{\mathbf{Q}^{i}(x)}+\mathbf{P}^{i}(x) \ln \frac{\pi^{i}}{\gamma^{i}}\right) \\
& =\sum_{i} \pi^{i} \mathrm{KL}\left(\mathbf{P}^{i} \| \mathbf{Q}^{i}\right)+\sum_{i} \pi^{i} \ln \frac{\pi^{i}}{\gamma^{i}} \\
& =\left(\sum_{i \in \mathcal{I}} \pi^{i} \operatorname{KL}\left(\mathbf{P}^{i} \| \mathbf{Q}^{i}\right)\right)+\left(\sum_{i \notin \mathcal{I}} \pi^{i} \mathrm{KL}\left(\mathbf{P}^{i} \| \mathbf{Q}^{i}\right)\right)+\sum_{i} \pi^{i} \ln \frac{\pi^{i}}{\gamma^{i}} .
\end{aligned}
$$

Copyright $@$ by SIAM. Unauthorized reproduction of this article is prohibited. 
For the first term of (7), we have

$$
\sum_{i \in \mathcal{I}} \pi^{i} \operatorname{KL}\left(\mathbf{P}^{i} \| \mathbf{Q}^{i}\right) \leq \epsilon_{\mathcal{I}}
$$

For the second term of (7), we have

$$
\sum_{i \notin \mathcal{I}} \pi^{i} \operatorname{KL}\left(\mathbf{P}^{i} \| \mathbf{Q}^{i}\right) \leq k \epsilon_{3} \cdot \max _{i \in[k]}\left\{\operatorname{KL}\left(\mathbf{P}^{i} \| \mathbf{Q}^{i}\right)\right\} \leq k \epsilon_{3} \epsilon_{\text {all }} .
$$

For the third term of $(7)$, letting $\mathcal{I}^{\prime}=\left\{i \in \mathcal{I}: \pi^{i} \geq \epsilon_{4}\right\}$, we have

$$
\sum_{i} \pi^{i} \ln \frac{\pi^{i}}{\gamma^{i}}=\sum_{i \notin \mathcal{I}^{\prime}} \pi^{i} \ln \frac{\pi^{i}}{\gamma^{i}}+\sum_{i \in \mathcal{I}^{\prime}} \pi^{i} \ln \frac{\pi^{i}}{\gamma^{i}} .
$$

For the first sum in (8) we have

$$
\sum_{i \notin \mathcal{I}^{\prime}} \pi^{i} \ln \frac{\pi^{i}}{\gamma^{i}} \leq k \epsilon_{4} \ln \frac{\epsilon_{4}}{\epsilon_{2}}
$$

For the second sum in (8), note first that for any $i$ such that $\pi^{i}<\gamma^{i}$, the contribution to the second sum is negative. For any other $i$, we have $\pi^{i} \geq \gamma^{i}$ and therefore $\gamma^{i} \geq \pi^{i}-\epsilon_{1}$. Consequently we have $\frac{\pi^{i}}{\gamma^{i}} \leq \frac{\pi^{i}}{\pi^{i}-\epsilon_{1}}=1+\frac{\epsilon_{1}}{\pi^{i}-\epsilon_{1}} \leq 1+\frac{\epsilon_{1}}{\epsilon_{4}-\epsilon_{1}}$. Hence the second sum in (8) is at most

$$
\sum_{i \in \mathcal{I}^{\prime}} \pi^{i} \ln \frac{\pi^{i}}{\gamma^{i}} \leq \sum_{i \in \mathcal{I}^{\prime}} \pi^{i} \ln \left(1+\frac{\epsilon_{1}}{\epsilon_{4}-\epsilon_{1}}\right) \leq \frac{\epsilon_{1}}{\epsilon_{4}-\epsilon_{1}} .
$$

Putting all the bounds together, the proof is done.

Finally, we will also need the following elementary proposition.

Proposition 14. Let $\mathbf{P}$ and $\mathbf{Q}$ denote distributions over $\{0, \ldots, b-1\}$, where $\mathbf{P}$ has probabilities $p_{0}, \ldots, p_{b-1}$ and $\mathbf{Q}$ has probabilities $q_{0}, \ldots, q_{b-1}$. Suppose that $\left|p_{\ell}-q_{\ell}\right|<\xi \leq \frac{1}{4}$ for all $\ell \in\{0, \ldots, b-1\}$, and that also $q_{\ell} \geq \tau$ for all $\ell \in\{0, \ldots, b-1\}$, where $\tau<\xi$. Then $\operatorname{KL}(\mathbf{P} \| \mathbf{Q}) \leq 2 \xi^{1 / 2}+b \xi^{3 / 2} / \tau$.

Proof. Let $L_{\text {small }}=\left\{\ell \in\{0, \ldots, b-1\}: p_{\ell} \leq \xi^{1 / 2}\right\}$ and $L_{b i g}=\{0, \ldots, b-1\} \backslash$ $L_{\text {small }}$. We bound the contribution to $\mathrm{KL}(\mathbf{P} \| \mathbf{Q})$ from $L_{\text {small }}$ and $L_{\text {big }}$ separately.

Now for the $L_{\text {small }}$ case. For all $\ell$, it is easy to see that $\ln \frac{p_{\ell}}{q_{\ell}} \leq \ln \frac{\xi+\tau}{\tau}=\ln \left(1+\frac{\xi}{\tau}\right) \leq$ $\frac{\xi}{\tau}$. Thus each $\ell \in L_{\text {small }}$ contributes at most $p_{\ell} \ln \frac{p_{\ell}}{q_{\ell}} \leq \frac{\xi^{3 / 2}}{\tau}$. Since $\left|L_{\text {small }}\right| \leq b$, the total contribution to $\mathrm{KL}(\mathbf{P} \| \mathbf{Q})$ from $L_{\text {small }}$ is at most $b \frac{\xi^{3 / 2}}{\tau}$.

If $\ell \in L_{b i g}$, then we have

$$
\frac{p_{\ell}}{q_{\ell}} \leq \frac{p_{\ell}}{p_{\ell}-\xi}=1+\frac{\xi}{p_{\ell}-\xi} \leq 1+\frac{\xi}{\xi^{1 / 2}-\xi} \leq 1+2 \xi^{1 / 2},
$$

where the last inequality holds since $\xi^{1 / 2} \leq \xi^{1 / 2} / 2$ (since $\xi \leq \frac{1}{4}$ ). We thus have that the total contribution to $\mathrm{KL}(\mathbf{P} \| \mathbf{Q})$ from $\ell \in L_{b i g}$ is at most $\ln \left(1+2 \xi^{1 / 2}\right) \leq 2 \xi^{1 / 2}$. This proves the proposition. 
7.2. Proof of Theorem 11. We construct a mixture $\dot{\mathbf{Z}}$ of product distributions $\dot{\mathbf{Z}}^{1}, \ldots, \dot{\mathbf{Z}}^{k}$ by defining new mixing weights $\dot{\pi}^{i}$ and probabilities $\dot{p}_{j, \ell}^{i}$. The procedure $\mathcal{A}$ is defined as follows:

1. For all $i=1, \ldots, k$ let

$$
\ddot{\pi}^{i}= \begin{cases}\hat{\pi}^{i} & \text { if } \hat{\pi}^{i} \geq \epsilon_{\mathrm{wts}} \\ \epsilon_{\mathrm{wts}} & \text { if } \hat{\pi}^{i}<\epsilon_{\mathrm{wts}} .\end{cases}
$$

Now let $s$ be such that $s \sum_{i=1}^{k} \ddot{\pi}^{i}=1$, and take $\dot{\pi}^{i}=s \ddot{\pi}^{i}$.

2. For all $i \in[k]$ and $j \in[n]$, let

$$
\ddot{p}_{j, \ell}^{i}= \begin{cases}\hat{p}_{j, \ell}^{i} & \text { if } \hat{p}_{j, \ell}^{i} \geq \epsilon_{\mathrm{bprobs}} \\ \epsilon_{\mathrm{bprobs}} & \text { if } \hat{p}_{j, \ell}^{i}<\epsilon_{\mathrm{bprobs}}\end{cases}
$$

Now let $t$ be such that $t \sum_{\ell \in\{0, \ldots, b-1\}} \ddot{p}_{j, \ell}^{i}=1$, and take $\dot{p}_{j, \ell}^{i}=t \ddot{p}_{j, \ell}^{i}$.

It is clear from construction that this yields $\dot{\pi}^{i}, \dot{p}_{j, \ell}^{i}$ that satisfy condition 1 (a) of the theorem. It is also clear that for each $i \in[k]$ we have that the distribution $\dot{\mathbf{Z}}^{i}$ satisfies $\dot{\mathbf{Z}}^{i}(x) \geq \epsilon_{\text {bprobs }}^{n}$ for all $x \in\{0, \ldots, b-1\}^{n}$, and thus the mixture $\dot{\mathbf{Z}}$ must satisfy $\dot{\mathbf{Z}}(x) \geq \epsilon_{\mathrm{bprobs}}^{n}$ for all these $x$. This gives part $1(\mathrm{~b})$ of the theorem.

We now turn to part 2, and henceforth assume that the conditions on $\pi^{i}, \hat{\pi}^{i}, p_{j, \ell}^{i}$, $\hat{p}_{j, \ell}^{i}$ from part 2 are indeed all satisfied. Roughly speaking, these conditions tell us that $\hat{\pi}^{i}, \hat{p}_{j, \ell}^{i}$ are "good" (in the sense that they are parametrically accurate); we will show that the resulting $\dot{\pi}^{i}, \dot{p}_{j, \ell}^{i}$ are "good" (in the sense of giving rise to a mixture $\dot{\mathbf{Z}}$ that satisfies (6)).

Our goal is to apply Proposition 13 with parameter settings

$$
\begin{array}{r}
\epsilon_{1}=3 k \epsilon_{\mathrm{wts}}, \quad \epsilon_{2}=\frac{\epsilon_{\mathrm{wts}}}{2}, \quad \epsilon_{3}=\epsilon_{\text {minwt }}, \\
\epsilon_{4}=\epsilon_{\mathrm{wts}}^{1 / 2}, \quad \epsilon_{\mathcal{I}}=12 n b^{3} \epsilon_{\mathrm{bprobs}}^{1 / 2}, \quad \epsilon_{\mathrm{all}}=n \ln \left(b / \epsilon_{\mathrm{bprobs}}\right)
\end{array}
$$

to bound $\operatorname{KL}(\mathbf{Z} \| \dot{\mathbf{Z}})$. To satisfy the conditions of Proposition 13 we must (1) upper bound $\left|\pi^{i}-\dot{\pi}^{i}\right|$ for all $i$, (2) lower bound $\dot{\pi}^{i}$ for all $i$, (3) upper bound $\operatorname{KL}\left(\mathbf{Z}^{i} \| \dot{\mathbf{Z}}^{i}\right)$ for all $i$ such that $\pi^{i} \geq \epsilon_{\text {minwt }}$, and (4) upper bound $\operatorname{KL}\left(\mathbf{Z}^{i} \| \dot{\mathbf{Z}}^{i}\right)$ for all $i \in[k]$. We now do this.

(1) Upper bounding $\left|\pi^{i}-\dot{\pi}^{i}\right|$. Fix any $i \in[k]$. If $\hat{\pi}^{i} \geq \epsilon_{\mathrm{wts}}$, then we have $\ddot{\pi}^{i}=\hat{\pi}^{i}$, so $\left|\pi^{i}-\ddot{\pi}^{i}\right| \leq \epsilon_{\mathrm{wts}}$. On the other hand, if $\hat{\pi}^{i}<\epsilon_{\mathrm{wts}}$, then it must be the case that $\pi^{i} \leq 2 \epsilon_{\mathrm{wts}}$, so we again have $\left|\pi^{i}-\ddot{\pi}^{i}\right| \leq \epsilon_{\mathrm{wts}}$. Since $\sum_{i=1}^{k} \pi^{i}=1$ it follows that

$$
\left|\sum_{i=1}^{k} \ddot{\pi}^{i}-1\right| \leq k \epsilon_{\mathrm{wts}}
$$

and thus

$$
\sum_{i=1}^{k} \ddot{\pi}^{i} \in\left[1-k \epsilon_{\mathrm{wts}}, 1+k \epsilon_{\mathrm{wts}}\right] .
$$

By definition of $s$ this gives

$$
s \in\left[\frac{1}{1+k \epsilon_{\mathrm{wts}}}, \frac{1}{1-k \epsilon_{\mathrm{wts}}}\right] .
$$

Copyright $@$ by SIAM. Unauthorized reproduction of this article is prohibited. 
Multiplying inequality (10) by $s$, recalling that $s \sum_{i=1}^{k} \ddot{\pi}^{i}=1$, and assuming $\epsilon_{\mathrm{wts}} \leq$ $1 /(2 k)$, we obtain

$$
|1-s| \leq s k \epsilon_{\mathrm{wts}} \leq \frac{k \epsilon_{\mathrm{wts}}}{1-k \epsilon_{\mathrm{wts}}} \leq 2 k \epsilon_{\mathrm{wts}}
$$

Thus, we have

$$
\begin{aligned}
\left|\pi^{i}-\dot{\pi}^{i}\right| & \leq\left|\pi^{i}-\ddot{\pi}^{i}\right|+\left|\ddot{\pi}^{i}-\dot{\pi}^{i}\right| \\
& \leq \epsilon_{\mathrm{wts}}+\left|\ddot{\pi}^{i}-\dot{\pi}^{i}\right| \\
& =\epsilon_{\mathrm{wts}}+\left|(1-s) \ddot{\pi}^{i}\right| \\
& \leq \epsilon_{\mathrm{wts}}+2 k \epsilon_{\mathrm{wts}}\left|\ddot{\pi}^{i}\right| \\
& \leq \epsilon_{\mathrm{wts}}+2 k \epsilon_{\mathrm{wts}} ;
\end{aligned}
$$

certainly, this gives $\left|\pi^{i}-\dot{\pi}^{i}\right| \leq 3 k \epsilon_{\mathrm{wts}}$.

(2) Lower bounding $\dot{\pi}^{i}$. To lower bound $\dot{\pi}^{i}$, we note that since $\ddot{\pi}^{i} \geq \epsilon_{\mathrm{wts}}$ for all $i$, under the assumption $\epsilon_{\mathrm{wts}} \leq 1 /(2 k)$, we have

$$
\dot{\pi}^{i}=s \ddot{\pi}^{i} \geq \frac{1}{1+k \epsilon_{\mathrm{wts}}} \ddot{\pi}^{i} \geq \frac{\epsilon_{\mathrm{wts}}}{1+k \epsilon_{\mathrm{wts}}} \geq \frac{2 \epsilon_{\mathrm{wts}}}{3},
$$

where the first inequality follows from (11).

(3) Upper bounding $\operatorname{KL}\left(\mathbf{Z}^{i} \| \dot{\mathbf{Z}}^{i}\right)$ for all $i$ such that $\pi^{i} \geq \epsilon_{\text {minwt. }}$. Fix an $i$ such that $\pi^{i} \geq \epsilon_{\text {minwt }}$, and fix any $j \in[n]$. Let $\mathbf{P}$ denote the distribution over $\{0, \ldots, b-1\}$ with probabilities $p_{j, 0}^{i}, \ldots, p_{j, b-1}^{i}$, and let $\mathbf{Q}$ denote the distribution over $\{0, \ldots, b-1\}$ with probabilities $\dot{p}_{j, 0}^{i}, \ldots, \dot{p}_{j, b-1}^{i}$.

We first show that each $\dot{p}_{j, \ell}^{i}$ is close to $\hat{p}_{j, \ell}^{i}$ and thus also to $p_{j, \ell}^{i}$. This is done very much as in (1) above. If $\hat{p}_{j, \ell}^{i} \geq \epsilon_{\mathrm{bprobs}}$, then we have $\ddot{p}_{j, \ell}^{i}=\hat{p}_{j, \ell}^{i}$, and so $\left|p_{j, \ell}^{i}-\ddot{p}_{j, \ell}^{i}\right| \leq$ $\epsilon_{\text {bprobs }}$ (by condition 2(b) in the theorem statement). On the other hand, if $\hat{p}_{j, \ell}^{i}<$ $\epsilon_{\text {bprobs }}$, then it must be the case that $p_{j, \ell}^{i} \leq 2 \epsilon_{\text {bprobs }}$, so we again have $\left|p_{j, \ell}^{i}-\ddot{p}_{j, \ell}^{i}\right| \leq$ $\epsilon_{\text {bprobs. }}$. Since $\sum_{\ell=0}^{b-1} p_{j, \ell}^{i}=1$ it follows that

$$
\left|\sum_{\ell=0}^{b-1} \ddot{p}_{j, \ell}^{i}-1\right| \leq b \epsilon_{\mathrm{bprobs}}
$$

and thus

$$
\sum_{\ell=0}^{b-1} \ddot{p}_{j, \ell}^{i} \in\left[1-b \epsilon_{\mathrm{bprobs}}, 1+b \epsilon_{\mathrm{bprobs}}\right] .
$$

By definition of $t$ this gives

$$
t \in\left[\frac{1}{1+b \epsilon_{\mathrm{bprobs}}}, \frac{1}{1-b \epsilon_{\mathrm{bprobs}}}\right] .
$$

Multiplying inequality (12) by $t$, recalling that $t \sum_{\ell=0}^{b-1} \ddot{p}_{j, \ell}^{i}=1$, and assuming $\epsilon_{\mathrm{bprobs}} \leq$ $1 /(2 b)$, we obtain

$$
|1-t| \leq t b \epsilon_{\mathrm{bprobs}} \leq \frac{b \epsilon_{\mathrm{bprobs}}}{1-b \epsilon_{\mathrm{bprobs}}} \leq 2 b \epsilon_{\mathrm{bprobs}}
$$

Copyright $@$ by SIAM. Unauthorized reproduction of this article is prohibited. 
Thus, we have

$$
\begin{aligned}
\left|p_{j, \ell}^{i}-\dot{p}_{j, \ell}^{i}\right| & \leq\left|p_{j, \ell}^{i}-\ddot{p}_{j, \ell}^{i}\right|+\left|\ddot{p}_{j, \ell}^{i}-\dot{p}_{j, \ell}^{i}\right| \\
& \leq \epsilon_{\mathrm{bprobs}}+\left|\ddot{p}_{j, \ell}^{i}-\dot{p}_{j, \ell}^{i}\right| \\
& =\epsilon_{\mathrm{bprobs}}+\left|(1-t) \ddot{p}_{j, \ell}^{i}\right| \\
& \leq \epsilon_{\mathrm{bprobs}}+2 b \epsilon_{\mathrm{bprobs}}\left|\ddot{p}_{j, \ell}^{i}\right| \\
& \leq \epsilon_{\mathrm{bprobs}}+2 b \epsilon_{\mathrm{bprobs}} ;
\end{aligned}
$$

certainly, this gives $\left|p_{j, \ell}^{i}-\dot{p}_{j, \ell}^{i}\right| \leq 3 b \epsilon_{\mathrm{bprobs}}$.

Moreover, since $\ddot{p}_{j, \ell}^{i} \geq \epsilon_{\mathrm{bprobs}}$ for all $\ell$ and $\dot{p}_{j, \ell}^{i}=t \ddot{p}_{j, \ell}^{i}$, where $t>\frac{1}{2}$ (by (13) and $\left.\epsilon_{\text {bprobs }} \leq 1 / b\right)$, we also have $\dot{p}_{j, \ell}^{i} \geq \epsilon_{\text {bprobs }} / 2$. We may thus apply Proposition 14 to $\mathbf{P}$ and $\mathbf{Q}$ (taking $\tau=\epsilon_{\text {bprobs }} / 2$ and $\xi=3 b \epsilon_{\text {bprobs }}$ ), and we obtain $\operatorname{KL}(\mathbf{P} \| \mathbf{Q}) \leq$ $2\left(3 b \epsilon_{\mathrm{bprobs}}\right)^{1 / 2}+b\left(3 b \epsilon_{\mathrm{bprobs}}\right)^{3 / 2} /\left(\epsilon_{\mathrm{bprobs}} / 2\right)$. A rough estimation gives that this is at

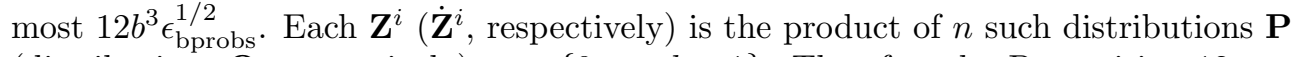
(distributions $\mathbf{Q}$, respectively) over $\{0, \ldots, b-1\}$. Therefore, by Proposition 12 , we have $\operatorname{KL}\left(\mathbf{Z}^{i} \| \dot{\mathbf{Z}}^{i}\right) \leq n \cdot\left(12 b^{3} \epsilon_{\text {bprobs }}^{1 / 2}\right)$ for all $i$ with $\pi^{i} \geq \epsilon_{\text {minwt }}$.

(4) Upper bounding $\operatorname{KL}\left(\mathbf{Z}^{i} \| \dot{\mathbf{Z}}^{i}\right)$ for all $i \in[k]$. This is simple: fix any $i \in[k]$. Since we know that $\dot{\mathbf{Z}}^{i}(x) \geq \epsilon_{\mathrm{bprobs}}^{n}$ for all $x \in\{0, \ldots, b-1\}^{n}$, we immediately have

$$
\mathrm{KL}\left(\mathbf{Z}^{i} \| \dot{\mathbf{Z}}^{i}\right) \leq-H\left(\mathbf{Z}^{i}\right)+\ln \left(1 /\left(\epsilon_{\text {bprobs }}\right)^{n}\right) \leq n \ln \left(b / \epsilon_{\text {bprobs }}\right),
$$

where $H(\mathbf{X}):=\sum_{x} \mathbf{X}(x) \ln (1 / \mathbf{X}(x))$ denotes the "entropy in nats" of the random variable $\mathbf{X}$.

We can now apply Proposition 13 with the parameter settings given by (9). Proposition 13 implies

$$
\begin{aligned}
\mathrm{KL}(\mathbf{Z} \| \dot{\mathbf{Z}}) \leq n \cdot\left(12 b^{3} \epsilon_{\mathrm{bprobs}}^{1 / 2}\right) & +k \epsilon_{\mathrm{minwt}} n \ln \left(b / \epsilon_{\mathrm{bprobs}}\right) \\
+ & {\left[k \epsilon_{\mathrm{wts}}^{1 / 2} \ln \frac{\epsilon_{\mathrm{wts}}^{1 / 2}}{\epsilon_{\mathrm{wts}} / 2}+\frac{3 k \epsilon_{\mathrm{wts}}}{\epsilon_{\mathrm{wts}}^{1 / 2}-3 k \epsilon_{\mathrm{wts}}}\right] . }
\end{aligned}
$$

Considering the terms of the expression in brackets above, if we set $\epsilon_{\mathrm{wts}}=\frac{c}{k^{7}}$ for some appropriately chosen small constant $c$, then we have that

$$
k \epsilon_{\mathrm{wts}}^{1 / 2} \ln \frac{\epsilon_{\mathrm{wts}}^{1 / 2}}{\epsilon_{\mathrm{wts}} / 2}=k \epsilon_{\mathrm{wts}}^{1 / 2} \ln \frac{2}{\epsilon_{\mathrm{wts}}^{1 / 2}} \leq \frac{1}{2} \epsilon_{\mathrm{wts}}^{1 / 3}
$$

and

$$
\frac{3 k \epsilon_{\mathrm{wts}}}{\epsilon_{\mathrm{wts}}^{1 / 2}-3 k \epsilon_{\mathrm{wts}}} \leq 6 k \epsilon_{\mathrm{wts}}^{1 / 2} \leq \frac{1}{2} \epsilon_{\mathrm{wts}}^{1 / 3}
$$

Hence

$$
\mathrm{KL}(\mathbf{Z} \| \dot{\mathbf{Z}}) \leq n \cdot\left(12 b^{3} \epsilon_{\text {bprobs }}^{1 / 2}\right)+k \epsilon_{\text {minwt }} n \ln \left(b / \epsilon_{\text {bprobs }}\right)+\epsilon_{\mathrm{wts}}^{1 / 3} .
$$

This concludes the proof of Theorem 11 .

Copyright $@$ by SIAM. Unauthorized reproduction of this article is prohibited. 
7.3. Some candidate distribution is good. Here we establish the following.

THEOREM 15. Let $b=O(1)$, and let $\mathbf{Z}$ be any unknown mixture of $k$ product distributions over $\{0, \ldots, b-1\}^{n}$. There is a poly $(n / \epsilon) \cdot \log \frac{1}{\delta}$ time algorithm which, given samples from $\mathbf{Z}$, outputs a list of poly $(n / \epsilon)$ many mixtures of product distributions over $\{0, \ldots, b-1\}^{n}$ with the properties that

1. every distribution $\mathbf{Z}^{\prime}$ in the list satisfies $\left(\frac{\epsilon}{36 n b^{3}}\right)^{2 n} \leq \mathbf{Z}^{\prime}(x) \leq 1$ for all $x \in$ $\{0, \ldots, b-1\}^{n} ;$ and

2. with probability $1-\delta$, some distribution $\mathbf{Z}^{\star}$ in the list satisfies $\mathrm{KL}\left(\mathbf{Z} \| \mathbf{Z}^{\star}\right) \leq \epsilon$.

Proof. We will use a specialization of Theorem 10 in which we have different parameters for the different roles that $\epsilon$ plays, as follows.

TheOREM $10^{\prime}$. Fix $k=O(1), b=O(1)$. Let $\mathbf{Z}$ be a mixture of $k$ product distributions $\mathbf{X}^{1}, \ldots, \mathbf{X}^{k}$ over $\{0, \ldots, b-1\}^{n}$, so that $\mathbf{Z}$ is described by mixing weights $\pi^{1}, \ldots, \pi^{k}$ and probabilities $\left\{p_{j, \ell}^{i}\right\}_{i \in[k], j \in[n], \ell \in\{0, \ldots, b-1\}}$.

There is an algorithm with the following property: for any $\epsilon_{\mathrm{wts}}, \epsilon_{\mathrm{bprobs}}, \epsilon_{\mathrm{minwt}}, \delta>$ 0 , with probability $1-\delta$ the algorithm outputs a list of candidates $\left\langle\left\{\hat{\pi}^{i}\right\},\left\{\hat{p}_{j, \ell}^{i}\right\}\right\rangle$ such that for at least one candidate in the list, the following hold:

1. $\left|\hat{\pi}^{i}-\pi^{i}\right| \leq \epsilon_{\mathrm{wts}}$ for all $i \in[k]$; and

2. $\left|\hat{p}_{j, \ell}^{i}-p_{j, \ell}^{i}\right| \leq \epsilon_{\mathrm{bprobs}}$ for all $i, j, \ell$ such that $\pi^{i} \geq \epsilon_{\mathrm{minwt}}$. The algorithm runs in time poly $\left(n / \epsilon^{\prime}\right) \cdot \log (1 / \delta)$, where $\epsilon^{\prime}=\min \left\{\epsilon_{\mathrm{wts}}, \epsilon_{\mathrm{bprobs}}, \epsilon_{\mathrm{minwt}}\right\}$.

Let $\epsilon, \delta>0$ be given. We run the algorithm of Theorem $10^{\prime}$ with parameters $\epsilon_{\text {bprobs }}=\left(\frac{\epsilon}{36 n b^{3}}\right)^{2}, \epsilon_{\text {minwt }}=\frac{\epsilon}{3 k n \ln \left(1296 b^{7} n^{2} / \epsilon^{2}\right)}$, and $\epsilon_{\mathrm{wts}}=\frac{\epsilon^{3}}{27}$. With these parameters the algorithm runs in time poly $(n / \epsilon) \cdot \log \frac{1}{\delta}$. By Theorem $10^{\prime}$, we get as output a list of $\operatorname{poly}(n / \epsilon)$ many candidate parameter settings $\left\langle\left\{\hat{\pi}^{i}\right\},\left\{\hat{\mu}_{j}^{i}\right\}\right\rangle$ with the guarantee that with probability $1-\delta$ at least one of the settings satisfies

- $\left|\pi^{i}-\hat{\pi}^{i}\right| \leq \epsilon_{\mathrm{wts}}$ for all $i \in[k]$, and

- $\left|\hat{p}_{j, \ell}^{i}-p_{j, \ell}^{i}\right| \leq \epsilon_{\mathrm{bprobs}}$ for all $i, j, \ell$ such that $\pi^{i} \geq \epsilon_{\text {minwt }}$.

We now pass each of these candidate parameter settings through Theorem 11. It follows that the resulting distributions each satisfy $\epsilon_{\mathrm{bprobs}}^{n}=\left(\frac{\epsilon}{36 n b^{3}}\right)^{2 n} \leq \mathbf{Z}^{\prime}(x) \leq 1$ for all $x \in\{0,1\}^{n}$. A routine verification shows that with our choice of $\epsilon_{\mathrm{bprobs}}, \epsilon_{\text {minwt }}$, and $\epsilon_{\mathrm{wts}}$ we have

$$
n \cdot\left(12 b^{3} \epsilon_{\mathrm{bprobs}}^{1 / 2}\right) \leq \frac{\epsilon}{3}, \quad k \epsilon_{\mathrm{minwt}} n \ln \frac{b}{\epsilon_{\mathrm{bprobs}}} \leq \frac{\epsilon}{3}, \quad \text { and } \quad \epsilon_{\mathrm{wts}}^{1 / 3} \leq \frac{\epsilon}{3} .
$$

Thus $\eta\left(\epsilon_{\mathrm{bprobs}}, \epsilon_{\mathrm{wts}}, \epsilon_{\mathrm{minwt}}\right) \leq \epsilon$, and we have that at least one of the resulting distributions $\mathbf{Z}^{\star}$ satisfies $\operatorname{KL}\left(\mathbf{Z}|| \mathbf{Z}^{\star}\right) \leq \epsilon$.

8. Finding a good hypothesis using maximum likelihood. Theorem 15 gives us a list of distributions, at least one of which is close to the target mixture distribution $\mathbf{Z}$ that we are trying to learn. Now we must identify some distribution in the list which is close to the target. In this section we give a simple maximum likelihood algorithm which helps us accomplish this. This is a standard situation (see, e.g., section 4.6 of [14]), and we emphasize that the ideas behind Theorem 16 below are not new. However, we were unable to find in the literature a clear statement of the exact result which we need, so for completeness we give our own statement and proof below.

Let $\mathbf{P}$ be a target distribution over some space $X$. Let $\mathcal{Q}$ be a set of hypothesis distributions such that at least one $\mathbf{Q}^{*} \in \mathcal{Q}$ has $\mathrm{KL}\left(\mathbf{P} \| \mathbf{Q}^{*}\right) \leq \epsilon$. The following algorithm will be used to find a distribution $\mathbf{Q}^{\mathrm{ML}} \in \mathcal{Q}$ which is close to $\mathbf{P}$. Draw a set 
$\mathcal{S}$ of samples from the distribution $\mathbf{P}$. For each $\mathbf{Q} \in \mathcal{Q}$, compute the log-likelihood

$$
\Lambda(\mathbf{Q})=\sum_{x \in \mathcal{S}}(-\ln \mathbf{Q}(x)) .
$$

Now output the distribution $\mathbf{Q}^{\mathrm{ML}} \in \mathcal{Q}$ such that $\Lambda(\mathbf{Q})$ is minimum. This is known as the maximum likelihood (ML) algorithm since it outputs the distribution in $\mathcal{Q}$ which maximizes $\arg \max _{\mathbf{Q} \in \mathcal{Q}} \prod_{x \in S} \mathbf{Q}(x)$.

THEOREM 16. Let $\beta, \alpha, \epsilon>0$ be such that $\alpha<\beta$. Let $\mathcal{Q}$ be a set of hypothesis distributions for some distribution $\mathbf{P}$ over the space $X$ such that at least one $\mathbf{Q}^{*} \in \mathcal{Q}$ has $\mathrm{KL}\left(\mathbf{P} \| \mathbf{Q}^{*}\right) \leq \epsilon$. Suppose also that $\alpha \leq \mathbf{Q}(x) \leq \beta$ for all $\mathbf{Q} \in \mathcal{Q}$ and all $x$ such that $\mathbf{P}(x)>0$.

Run the $M L$ algorithm on $\mathcal{Q}$ using a set $\mathcal{S}$ of independent samples from $\mathbf{P}$, where $|\mathcal{S}|=m$. Then, with probability $1-\delta$, where

$$
\delta \leq(|\mathcal{Q}|+1) \cdot \exp \left(-2 m \frac{\epsilon^{2}}{\log ^{2}(\beta / \alpha)}\right)
$$

the algorithm outputs some distribution $\mathbf{Q}^{\mathrm{ML}} \in \mathcal{Q}$ which has $\mathrm{KL}\left(\mathbf{P} \| \mathbf{Q}^{\mathrm{ML}}\right) \leq 4 \epsilon$.

Before proving Theorem 16 we give some preliminaries. Let $\mathbf{P}$ and $\mathbf{Q}$ be arbitrary distributions over some space $X$. We can rewrite the KL divergence between $\mathbf{P}$ and Q as

$$
\mathrm{KL}(\mathbf{P} \| \mathbf{Q})=-H(\mathbf{P})-\sum_{x \in X} \mathbf{P}(x) \ln \mathbf{Q}(x),
$$

where $H(\mathbf{P})=-\sum_{x \in X} \mathbf{P}(x) \ln \mathbf{P}(x)$ is the "entropy in nats" of $\mathbf{P}$.

Consider the random variable $-\ln \mathbf{Q}(x)$, where $x$ is a sample from the distribution P. Using (14), we can express the expectation of this variable in terms of the KL divergence:

$$
\mathbf{E}_{x \in \mathbf{P}}[-\ln \mathbf{Q}(x)]=\operatorname{KL}(\mathbf{P} \| \mathbf{Q})+H(\mathbf{P}) .
$$

Recall that when the ML algorithm runs on a list $\mathcal{Q}$ of distributions, it uses a set $\mathcal{S}$ of independent samples from $\mathbf{P}$, where $m=|\mathcal{S}|$. For each distribution $\mathbf{Q} \in \mathcal{Q}$, the algorithm computes

$$
\Lambda(\mathbf{Q})=\sum_{x \in \mathcal{S}}(-\ln \mathbf{Q}(x))
$$

So, by (15), we have that the expected "score" of distribution $\mathbf{Q}$ is the following:

$$
\mathbf{E}_{\mathcal{S}}[\Lambda(\mathbf{Q})]=m(H(\mathbf{P})+\mathrm{KL}(\mathbf{P} \| \mathbf{Q})) .
$$

We recall the theorem of Hoeffding [16], as follows.

THEOREM 17 (Hoeffding). Let $x_{1}, \ldots, x_{n}$ be independent bounded random variables such that each $x_{i}$ falls into the interval $[a, b]$ with probability one. Let $X=$ $\sum_{i=1}^{n} x_{i}$. Then for any $t>0$ we have

$$
\operatorname{Pr}[X-\mathbf{E}[X] \geq t] \leq e^{-2 t^{2} / n(b-a)^{2}} \quad \text { and } \quad \operatorname{Pr}[X-\mathbf{E}[X] \leq-t] \leq e^{-2 t^{2} / n(b-a)^{2}} .
$$

Now we can prove Theorem 16. 
Proof of Theorem 16. Call a distribution $\mathbf{Q} \in \mathcal{Q}$ good if $\mathrm{KL}\left(\mathbf{P} \| \mathbf{Q}^{\mathrm{ML}}\right) \leq 4 \epsilon$, and bad otherwise. Note that, by assumption, we have at least one good distribution in $\mathcal{Q}$.

The probability $\delta$ that the algorithm fails to output some good distribution is at most the probability that either some bad distribution $\mathbf{Q}$ has $\Lambda(\mathbf{Q}) \leq m(H(\mathbf{P})+3 \epsilon)$ or the good distribution $\mathbf{Q}^{*}$ has $\Lambda\left(\mathbf{Q}^{*}\right) \geq m(H(\mathbf{P})+2 \epsilon)$. Thus, by a union bound, we have

$$
\begin{gathered}
\delta \leq|\mathcal{Q}| \cdot \operatorname{Pr}[\Lambda(\mathbf{Q}) \leq m(H(\mathbf{P})+3 \epsilon) \mid \operatorname{KL}(\mathbf{P} \| \mathbf{Q}) \geq 4 \epsilon] \\
+\operatorname{Pr}\left[\Lambda\left(\mathbf{Q}^{*}\right) \geq m(H(\mathbf{P})+2 \epsilon)\right] .
\end{gathered}
$$

For each bad $\mathbf{Q} \in \mathcal{Q}$ which has $\operatorname{KL}(\mathbf{P} \| \mathbf{Q})>4 \epsilon$ we have

$$
\begin{aligned}
\operatorname{Pr}[\Lambda(\mathbf{Q}) \leq m(H(\mathbf{P})+3 \epsilon)] & =\operatorname{Pr}[\Lambda(\mathbf{Q}) \leq m(H(\mathbf{P})+4 \epsilon)-\epsilon m)] \\
& \leq \operatorname{Pr}[\Lambda(\mathbf{Q}) \leq m(H(\mathbf{P})+\operatorname{KL}(\mathbf{P} \| \mathbf{Q}))-\epsilon m)] \\
& =\operatorname{Pr}\left[\Lambda(\mathbf{Q}) \leq \mathbf{E}_{\mathcal{S}}[\Lambda(\mathbf{Q})]-\epsilon m\right] \\
& \leq \exp \left(-2 m \frac{\epsilon^{2}}{\log ^{2}(\beta / \alpha)}\right) .
\end{aligned}
$$

Equation (18) follows from the bound on the KL divergence, (19) follows from (16), and (20) follows from the Hoeffding bound (Theorem 17).

Following the same logic for $\mathbf{Q}^{*}$ where $\operatorname{KL}\left(\mathbf{P} \| \mathbf{Q}^{*}\right) \leq \epsilon$, we get

$$
\begin{aligned}
\operatorname{Pr}\left[\Lambda\left(\mathbf{Q}^{*}\right) \geq m(H(\mathbf{P})+2 \epsilon)\right] & =\operatorname{Pr}\left[\Lambda\left(\mathbf{Q}^{*}\right) \geq m(H(\mathbf{P})+\epsilon)+m \epsilon\right] \\
& \leq \operatorname{Pr}\left[\Lambda\left(\mathbf{Q}^{*}\right) \geq m\left(H(\mathbf{P})+\operatorname{KL}\left(\mathbf{P} \| \mathbf{Q}^{*}\right)\right)+m \epsilon\right] \\
& =\operatorname{Pr}\left[\Lambda\left(\mathbf{Q}^{*}\right) \geq \mathbf{E}_{\mathcal{S}}\left[\Lambda\left(\mathbf{Q}^{*}\right)\right]+m \epsilon\right] \\
& \leq \exp \left(-2 m \frac{\epsilon^{2}}{\log ^{2}(\beta / \alpha)}\right) .
\end{aligned}
$$

Theorem 16 follows from plugging (20) and (21) into (17).

9. Putting it all together. All the pieces are now in place for us to prove our main learning result, Theorem 2, for learning mixtures of product distributions over $\{0, \ldots, b-1\}^{n}$.

Proof of Theorem 2. Run the algorithm described in Theorem 15. With probability $1-\delta$ this produces a list of $T=\operatorname{poly}(n / \epsilon)$ many hypothesis distributions, one of which has KL divergence at most $\epsilon$ from $\mathbf{Z}$ and each of which puts weight at least $\left(\frac{\epsilon}{36 n b^{3}}\right)^{2 n}$ on every point in $\{0, \ldots, b-1\}^{n}$. Now run the ML algorithm with $\alpha=\left(\frac{\epsilon}{36 n b^{3}}\right)^{2 n}, \beta=1$, and $m=\operatorname{poly}(n, 1 / \epsilon) \ln (T / \delta)$. By Theorem 16, with probability at least $1-\delta$ the ML algorithm outputs a hypothesis with KL divergence at most $4 \epsilon$ from $\mathbf{Z}$. Thus with overall probability $1-2 \delta$ we get a hypothesis with KL divergence at most $4 \epsilon$ from $\mathbf{Z}$, and the total running time is poly $(n / \epsilon) \cdot \log (1 / \delta)$. Replacing $\epsilon$ by $\epsilon / 4$ and $\delta$ by $\delta / 2$, we are done.

Tracing through the proofs, it is easy to check that the running time dependence on $k$ is $(n / \epsilon)^{O\left(k^{3}\right)} \cdot \log \frac{1}{\delta}$.

10. Hardness of learning mixtures of product distributions. In this section we give evidence that the class of mixtures of $k(n)$ product distributions over the Boolean cube may be hard to learn in polynomial time for any $k(n)=\omega(1)$.

Before describing our results, we recall some standard terminology about Boolean decision trees. A decision tree is a rooted binary tree in which each internal node has 
two children and is labeled with a variable and each leaf is labeled with a bit $b \in\{0,1\}$. A decision tree $T$ computes a Boolean function $f:\{0,1\}^{n} \rightarrow\{0,1\}$ in the obvious way: on input $x \in\{0,1\}^{n}$, if variable $x_{i}$ is at the root of $T$, we go to either the left or right subtree, depending on whether $x_{i}$ is 0 or 1 . Continue in this fashion until reaching a bit leaf; the value of this bit is $f(x)$.

Our main result in this section is the following theorem.

THEOREM 18. For any function $k(n)$, if there is a poly $(n / \epsilon)$-time algorithm which learns a mixture of $k(n)$ many product distributions over $\{0,1\}^{n}$, then there is a poly $(n / \epsilon)$ time uniform distribution PAC learning algorithm which learns the class of all $k(n)$-leaf decision trees.

We note that after years of intensive research, no poly $(n)$-time uniform distribution PAC learning algorithm is known which can learn $k(n)$-leaf decision trees for any $k(n)=\omega(1)$; indeed, such an algorithm would be a major breakthrough in computational learning theory. ${ }^{4}$ The fastest algorithms to date [12,3] can learn $k(n)$-leaf decision trees under the uniform distribution in time $n^{\log k(n)}$. This suggests that it may be impossible to learn mixtures of a superconstant number of product distributions over $\{0,1\}^{n}$ in polynomial time.

The basic idea behind this theorem is quite simple. Given any $k(n)$-leaf decision tree $T$, the set of all positive examples for $T$ is a union of at most $k(n)$ many disjoint subcubes of $\{0,1\}^{n}$, and thus the uniform distribution over the positive examples is a mixture of at most $k(n)$ product distributions over $\{0,1\}^{n}$. If we can obtain a high-accuracy hypothesis mixture $\mathcal{D}$ for this mixture of product distributions, then roughly speaking $\mathcal{D}$ must put "large" weight on the positive examples and "small" weight on the negative examples. We can thus use $\mathcal{D}$ to make accurate predictions of $T$ 's value on new examples very simply as follows: given a new example $x$ to classify, we simply compute the probability weight that the hypothesis mixture $\mathcal{D}$ puts on $x$, and output 1 or 0 depending on whether this weight is large or small.

We now give the formal proof of Theorem 18. The following claim is used in the proof.

Claim 1. Let $T$ be a k-leaf decision tree, let $b \in\{-1,1\}$ be a bit, let $S=$ $\left\{x \in\{0,1\}^{n}: T(x)=b\right\}$, and let $\mathcal{U}_{S}$ denote the uniform distribution over $S$. Then $\mathcal{U}_{S}$ is a mixture of $k$ product distributions.

Proof. We show that $\mathcal{U}_{S}$ is a mixture of $\ell$ product distributions, where $\ell$ is the number of leaves in $T$ which are labeled with bit $b$. To see this, observe that the $k$ leaves of $T$ partition $\{0,1\}^{n}$ into $k$ disjoint subsets, each consisting of those $x \in\{0,1\}^{n}$ which reach the corresponding leaf. For a leaf at depth $d$ the corresponding subset is of size $2^{n-d}$ and consists of those $x \in\{0,1\}^{n}$ which satisfy the length- $d$ conjunction defined by the path from the root to that leaf. Thus, choosing a uniform element of $S$ can be performed by the following process: (i) choose a leaf whose label is $b$, where each leaf at depth $d$ is chosen with probability proportional to $1 / 2^{d}$; and then (ii) choose a uniform random example from the set of examples which satisfy the conjunction corresponding to that leaf. The uniform distribution over examples which satisfy a given conjunction is easily seen to be a product distribution $\mathbf{X}$ over $\{0,1\}^{n}$ in which $\mathbf{E}\left[\mathbf{X}_{i}\right] \in\left\{0, \frac{1}{2}, 1\right\}$ for all $i=1, \ldots, n$. It follows that the uniform distribution over $S$ is a mixture of $\ell$ product distributions of this sort.

Proof of Theorem 18. We suppose that we are given access to an oracle $\operatorname{EX}(T, \mathcal{U})$ which, at each invocation, supplies a labeled example $(x, T(x)) \in\{0,1\}^{n} \times\{0,1\}$,

\footnotetext{
${ }^{4}$ Avrim Blum has offered a $\$ 1000$ prize for solving a subproblem of the $k(n)=n$ case and a $\$ 500$ prize for a subproblem of the $k(n)=\log n$ case; see [4].
} 
where $x$ is chosen from the uniform distribution $\mathcal{U}$ over $\{0,1\}^{n}$ and $T$ is the unknown $k(n)$-leaf decision tree to be learned. We describe an efficient algorithm $A^{\prime}$ which with probability $1-\delta$ outputs a hypothesis $h:\{0,1\}^{n} \rightarrow\{0,1\}$ which satisfies $\operatorname{Pr}_{\mathcal{U}}[h(x) \neq T(x)] \leq \epsilon$. The algorithm $A^{\prime}$ uses as a subroutine an algorithm $A$ which learns a mixture of $k(n)$ product distributions. Let $M$ be the number of examples required by algorithm $A$ to learn an unknown mixture of $k(n)$ product distributions to $L_{1}$-norm accuracy $1-\frac{\epsilon}{2}$ and confidence $1-\frac{\delta}{3}$. Recall from section 1.1 that to learn to $L_{1}$-norm error $\epsilon$ it suffices to learn to KL divergence $\epsilon^{2}$, and thus we have that $M=\operatorname{poly}(n / \epsilon)$ by our assumption on the running time of $A$.

Algorithm $A^{\prime}$ works as follows:

1. Determine $b \in\{-1,1\}$ such that with probability $1-\frac{\delta}{3}$ tree $T$ outputs $b$ on at least $1 / 3$ of the inputs in $\{0,1\}^{n}$. Let $S$ denote $\left\{x \in\{0,1\}^{n}: T(x)=b\right\}$, and let $\mathcal{U}_{S}$ denote the uniform distribution over $S$.

2. Run algorithm $A$ using samples from the uniform distribution $\mathcal{U}_{S}$; simulate $\mathcal{U}_{S}$ by invoking $\operatorname{EX}(T, \mathcal{U})$, and using the only examples with labels $T(x)=b$. To be confident that algorithm $A$ receives at least $M$ examples from $\mathcal{U}_{S}$, we draw $\Theta(M \log (1 / \delta))$ examples from $\operatorname{EX}(T, \mathcal{U})$. Let $\mathcal{D}^{\prime}$ be the hypothesis distribution which is the output of $A$.

3. Output the hypothesis $h:\{0,1\}^{n} \rightarrow\{-1,1\}$, which is defined as follows: given $x$, if $\mathcal{D}^{\prime}(x) \leq \frac{1}{2 \cdot 2^{n}}$, then $h(x)=-b$; else $h(x)=b$.

We now verify the algorithm's correctness. Note first that step 1 can easily be performed by making $O\left(\log \frac{1}{\delta}\right)$ draws from $\operatorname{EX}(T, \mathcal{U})$ to obtain an empirical estimate of $\operatorname{Pr}_{\mathcal{U}}[T(x)=b]$. Assuming that $|S|$ is indeed at least $2^{n} / 3$, a simple Chernoff bound shows that $O\left(M \log \frac{1}{\delta}\right)$ draws from $\operatorname{EX}(T, \mathcal{U})$ suffice to obtain $M$ examples with label $b$ in step 2 with probability $1-\frac{\delta}{3}$. We run $A$ on examples generated by $\mathcal{U}_{S}$, which by Claim 1 is a mixture of $k$ product distributions. Consequently, with overall probability at least $1-\delta$ the hypothesis $\mathcal{D}^{\prime}$ generated in step 2 satisfies $\left\|D^{\prime}-\mathcal{U}_{S}\right\|_{1} \leq \frac{\epsilon}{2}$.

Now observe that the hypothesis $h$ in step 3 disagrees with $T$ on precisely those $x$ which either (i) belong to $S$ but have $\mathcal{D}^{\prime}(x)<\frac{1}{2 \cdot 2^{n}}$, or (ii) do not belong to $S$ but have $\mathcal{D}^{\prime}(x) \geq \frac{1}{22^{n}}$. Each $x$ of type (i) contributes at least $\frac{1}{2 \cdot 2^{n}}$ toward $\left\|\mathcal{D}^{\prime}-\mathcal{U}_{S}\right\|_{1}$ since $\mathcal{U}_{S}(x) \geq \frac{1}{2^{n}}$ for each $x \in S$. Each $x$ of type (ii) also incurs at least $\frac{1}{2 \cdot 2^{n}}$ toward $\left\|\mathcal{D}^{\prime}-\mathcal{U}_{S}\right\|_{1}$. Consequently, since $\left\|\mathcal{D}^{\prime}-\mathcal{U}_{S}\right\|_{1} \leq \frac{\epsilon}{2}$, there are at most $\epsilon 2^{n}$ points $x \in\{0,1\}^{n}$ on which $h$ is wrong. Thus, we have shown that, with probability at least $1-\delta$, the hypothesis $h$ is an $\epsilon$-accurate hypothesis for $T$ with respect to the uniform distribution, as desired.

Remark 2. We note that our reduction to decision tree learning in fact uses only quite restricted mixtures of product distributions in which (i) the mixture coefficients are proportional to powers of 2, (ii) the supports of the product distributions in the mixture are mutually disjoint, and (iii) each product distribution is a uniform distribution over some subcube of $\{0,1\}^{n}$ (equivalently, each product distribution has each $\left.\mathbf{E}\left[\mathbf{X}_{i}\right] \in\{-1,0,1\}\right)$. Thus, even this restricted class of mixtures of $k(n)$ product distributions is as hard to learn as $k(n)$-leaf decision trees.

Remark 3. The known results of Blum et al. [5] imply the following unconditional hardness result: the class of $k(n)$-leaf decision trees cannot be learned under the uniform distribution in time less than $n^{\log k(n)}$ in the model of learning from statistical queries.

A "statistical query" learning algorithm is allowed to obtain only statistical estimates (accurate to within some specified error tolerance) of properties of the distribution over pairs $(x, T(x))$, and does not have access to actual labeled examples $(x, T(x))$. 
The algorithm is "charged" more time for estimates with a higher precision guarantee; this is motivated by the fact that such high-precision estimates would normally be obtained, given access to random examples, by drawing a large sample and making an empirical estimate. (See [17] for a detailed description of the statistical query model.)

Note that our algorithm for learning mixtures of product distributions interacts with the data solely by constructing empirical estimates of probabilities; thus, when this algorithm is used in the reduction of Theorem 18, the resulting algorithm for learning decision trees is easily seen to have an equivalent statistical query algorithm. Thus the results of Blum et al. unconditionally imply that no algorithm with the same basic approach as our algorithm can learn mixtures of $k(n)$ product distributions in time less than $n^{\log k(n)}$.

11. Conclusions and future work. We have shown how to learn mixtures of any constant number of product distributions over $\{0,1\}^{n}$, and more generally over $\{0, \ldots, b-1\}^{n}$, in polynomial time.

The methods we use are quite general and can be adapted to learn mixtures of other types of multivariate product distributions which are definable in terms of their moments. Along these lines, we have used the approach in this paper to give a PACstyle algorithm for learning mixtures of $k=O(1)$ axis-aligned Gaussians in polynomial time [13]. (We note that while some previous work on learning mixtures of Gaussians from a clustering perspective can handle $k=\omega(1)$ many component Gaussians, all such work assumes that there is some minimum separation between the centers of the component Gaussians, since otherwise clustering is clearly impossible. In contrast, our result in [13] - in which we do not attempt to do clustering but instead find a hypothesis distribution with small KL divergence from the target mixture - does not require us to assume that the component Gaussians are separated.) We expect that our techniques can also be adapted to learn mixtures of other distributions such as products of exponential distributions or beta distributions.

It is natural to ask whether our approach can be extended to learn mixtures of distributions which are not necessarily product distributions; this is an interesting direction for future work. Note that our main algorithmic ingredient, algorithm WAM, requires only that the coordinate distributions be pairwise independent.

Finally, one may also ask if it is possible to improve the efficiency of our learning algorithms - can the running times be reduced to $n^{O\left(k^{2}\right)}$, to $n^{O(k)}$, or even $n^{O(\log k)}$ ?

\section{REFERENCES}

[1] S. AARonson, Multilinear formulas and skepticism of quantum computation, in Proceedings of the 36th Annual Symposium on Theory of Computing (STOC), Chicago, IL, 2004, ACM, New York, pp. 118-127.

[2] S. Arora and R. Kannan, Learning mixtures of arbitrary Gaussians, in Proceedings of the 33rd Annual Symposium on Theory of Computing (STOC), Heraklion, Crete, 2001, ACM, New York, pp. 247-257.

[3] A. Blum, Rank-r decision trees are a subclass of $r$-decision lists, Inform. Process. Lett., 42 (1992), pp. 183-185.

[4] A. BLum, Learning a function of $r$ relevant variables (open problem), in Proceedings of the 16th Annual Conference on Learning Theory and 7th Kernel Workshop, Washington, DC, 2003, pp. 731-733.

[5] A. Blum, M. Furst, J. Jackson, M. Kearns, Y. Mansour, and S. Rudich, Weakly learning DNF and characterizing statistical query learning using Fourier analysis, in Proceedings of the 26th Annual Symposium on Theory of Computing (STOC), Montréal, QC, 1994, ACM, New York, pp. 253-262. 
[6] S. Chawla, C. Dwork, F. McSherry, A. Smith, and H. Wee, Towards privacy in public databases, in Proceedings of the 2nd Theory of Cryptography Conference (TCC), 2005, pp. $363-385$.

[7] T. Cover And J. Thomas, Elements of Information Theory, Wiley, New York, 1991.

[8] M. CRYAn, Learning and Approximation Algorithms for Problems Motivated by Evolutionary Trees, Ph.D. thesis, Computer Science, University of Warwick, Warwick, UK, 1999.

[9] M. Cryan, L. A. Goldberg, and P. W. Goldberg, Evolutionary trees can be learned in polynomial time in the two-state general Markov model, SIAM J. Comput., 31 (2001), pp. 375-397.

[10] S. DAsGupta, Learning mixtures of Gaussians, in Proceedings of the 40th Annual Symposium on Foundations of Computer Science (FOCS), New York, 1999, IEEE Computer Society Press, Piscataway, NJ, pp. 634-644.

[11] S. Dasgupta And L. Schulman, A two-round variant of EM for Gaussian mixtures, in Proceedings of the 16th Conference on Uncertainty in Artificial Intelligence, Seattle, WA, 2000, Morgan Kaufmann, San Francisco, pp. 143-151.

[12] A. Ehrenfeucht And D. Haussler, Learning decision trees from random examples, Inform. and Comput., 82 (1989), pp. 231-246.

[13] J. Feldman, R. O'Donnell, and R. Servedio, PAC learning mixtures of axis-aligned Gaussians with no separation assumption, in Proceedings of the 19th Annual Conference on Learning Theory (COLT), Pittsburgh, PA, 2006, Springer, New York, pp. 20-34; available online at http://arxiv.org/abs/cs.LG/0609093.

[14] Y. Freund And Y. Mansour, Estimating a mixture of two product distributions, in Proceedings of the 12th Annual Conference on Computational Learning Theory, Santa Cruz, CA, 1999, ACM, New York, pp. 183-192.

[15] S. Goreinov, E. Tyrtyshnikov, and N. Zamarashin, A theory of pseudoskeleton approximations, Linear Algebra Appl., 261 (1997), pp. 1-21.

[16] W. Hoeffoing, Probability inequalities for sums of bounded random variables, J. Amer. Statist. Assoc., 58 (1963), pp. 13-30.

[17] M. KeARns, Efficient noise-tolerant learning from statistical queries, J. ACM, 45 (1998), pp. 983-1006.

[18] M. Kearns, Y. Mansour, D. Ron, R. Rubinfeld, R. Schapire, and L. Sellie, On the learnability of discrete distributions, in Proceedings of the 26th Annual Symposium on Theory of Computing (STOC), Montréal, QC, 1994, ACM, New York, pp. 273-282.

[19] B. Lindsay, Mixture Models: Theory, Geometry, and Applications, Institute for Mathematical Statistics, Beachwood, OH, 1995.

[20] E. Mossel And S. Roch, Learning nonsingular phylogenies and hidden Markov models, in Proceedings of the 37th Annual Symposium on Theory of Computing (STOC), Baltimore, MD, 2005, pp. 366-375.

[21] R. A. Redner AND H. F. WALKer, Mixture densities, maximum likelihood, and the EM algorithm, SIAM Rev., 26 (1984), pp. 195-239.

[22] A. Smith, Personal communication, 2005.

[23] D. M. Titterington, A. F. M. Smith, and U. E. Makov, Statistical Analysis of Finite Mixture Distributions, Wiley, New York, 1985.

[24] S. Vempala And G. WANG, A spectral algorithm for learning mixtures of distributions, in Proceedings of the 43rd Annual Symposium on Foundations of Computer Science (FOCS), Vancouver, BC, 2002, IEEE Computer Society Press, Piscataway, NJ, pp. 113-122.

Copyright (c) by SIAM. Unauthorized reproduction of this article is prohibited. 